\section{Bregman weak relatively nonexpansive mappings in Banach spaces}

\author{
Eskandar Naraghirad ${ }^{1,2}$ and Jen-Chih $\mathrm{YaO}^{3,4^{*}}$
}

${ }^{\text {*Correspondence: }}$
yaojc@kmu.edu.tw
${ }^{3}$ Center for Fundamental Science,
Kaohsiung Medical University,
Kaohsiung, 807, Taiwan
${ }^{4}$ Department of Mathematics, King
Abdulaziz University, P.O. Box 80203 ,
Jeddah, 21589, Saudi Arabia
Full list of author information is
available at the end of the article

${ }^{\text {*Correspondence: }}$

yaojc@kmu.edu.tw

Center for Fundamental science,

Kaohsiung, 807, Taiwan

Jeddah, 21589, Saudi Arabia

available at the end of the article

\begin{abstract}
In this paper, we introduce a new class of mappings called Bregman weak relatively nonexpansive mappings and propose new hybrid iterative algorithms for finding common fixed points of an infinite family of such mappings in Banach spaces. We prove strong convergence theorems for the sequences produced by the methods. Furthermore, we apply our method to prove strong convergence theorems of iterative algorithms for finding common fixed points of finitely many Bregman weak relatively nonexpansive mappings in reflexive Banach spaces. These algorithms take into account possible computational errors. We also apply our main results to solve equilibrium problems in reflexive Banach spaces. Finally, we study hybrid iterative schemes for finding common solutions of an equilibrium problem, fixed points of an infinite family of Bregman weak relatively nonexpansive mappings and null spaces of a $\gamma$-inverse strongly monotone mapping in 2-uniformly convex Banach spaces. Some application of our results to the solution of equations of Hammerstein-type is presented. Our results improve and generalize many known results in the current literature.

MSC: $47 \mathrm{H} 10 ; 37 \mathrm{C} 25$
\end{abstract}

Keywords: Bregman function; uniformly convex function; uniformly smooth function; fixed point; strong convergence; Bregman weak relatively nonexpansive mapping

\section{Introduction}

The hybrid projection method was first introduced by Hangazeau in [1]. In a series of papers [2-12], authors investigated the hybrid projection method and proved strong and weak convergence theorems for the sequences produced by their method. The shrinking projection method, which is a generalization of the hybrid projection method, was first introduced by Takahashi $e t$ al. in [13]. Throughout this paper, we denote the set of real numbers and the set of positive integers by $\mathbb{R}$ and $\mathbb{N}$, respectively. Let $E$ be a Banach space with the norm $\|\cdot\|$ and the dual space $E^{*}$. For any $x \in E$, we denote the value of $x^{*} \in E^{*}$ at $x$ by $\left\langle x, x^{*}\right\rangle$. Let $\left\{x_{n}\right\}_{n \in \mathbb{N}}$ be a sequence in $E$. We denote the strong convergence of $\left\{x_{n}\right\}_{n \in \mathbb{N}}$ to $x \in E$ as $n \rightarrow \infty$ by $x_{n} \rightarrow x$ and the weak convergence by $x_{n} \rightarrow x$. The modulus $\delta$ of convexity of $E$ is denoted by

$$
\delta(\epsilon)=\inf \left\{1-\frac{\|x+y\|}{2}:\|x\| \leq 1,\|y\| \leq 1,\|x-y\| \geq \epsilon\right\}
$$

(O) 2013 Naraghirad and Yao; licensee Springer. This is an Open Access article distributed under the terms of the Creative Commons Attribution License (http://creativecommons.org/licenses/by/2.0), which permits unrestricted use, distribution, and reproduction in any medium, provided the original work is properly cited. 
for every $\epsilon$ with $0 \leq \epsilon \leq 2$. A Banach space $E$ is said to be uniformly convex if $\delta(\epsilon)>0$ for every $\epsilon>0$. Let $S_{E}=\{x \in E:\|x\|=1\}$. The norm of $E$ is said to be Gâteaux differentiable if for each $x, y \in S_{E}$, the limit

$$
\lim _{t \rightarrow 0} \frac{\|x+t y\|-\|x\|}{t}
$$

exists. In this case, $E$ is called smooth. If the limit (1.1) is attained uniformly for all $x, y \in$ $S_{E}$, then $E$ is called uniformly smooth. The Banach space $E$ is said to be strictly convex if $\left\|\frac{x+y}{2}\right\|<1$ whenever $x, y \in S_{E}$ and $x \neq y$. It is well known that $E$ is uniformly convex if and only if $E^{*}$ is uniformly smooth. It is also known that if $E$ is reflexive, then $E$ is strictly convex if and only if $E^{*}$ is smooth; for more details, see $[14,15]$.

Let $C$ be a nonempty subset of $E$. Let $T: C \rightarrow E$ be a mapping. We denote the set of fixed points of $T$ by $F(T)$, i.e., $F(T)=\{x \in C: T x=x\}$. A mapping $T: C \rightarrow E$ is said to be nonexpansive if $\|T x-T y\| \leq\|x-y\|$ for all $x, y \in C$. A mapping $T: C \rightarrow E$ is said to be quasi-nonexpansive if $F(T) \neq \emptyset$ and $\|T x-y\| \leq\|x-y\|$ for all $x \in C$ and $y \in F(T)$. The concept of nonexpansivity plays an important role in the study of Mann-type iteration [16] for finding fixed points of a mapping $T: C \rightarrow C$. Recall that the Mann-type iteration is given by the following formula:

$$
x_{n+1}=\gamma_{n} T x_{n}+\left(1-\gamma_{n}\right) x_{n}, \quad x_{1} \in C
$$

Here, $\left\{\gamma_{n}\right\}_{n \in \mathbb{N}}$ is a sequence of real numbers in $[0,1]$ satisfying some appropriate conditions. The construction of fixed points of nonexpansive mappings via Mann's algorithm [16] has been extensively investigated recently in the current literature (see, for example, [17] and the references therein). In [17], Reich proved the following interesting result.

Theorem 1.1 Let $C$ be a closed and convex subset of a uniformly convex Banach space $E$ with a Fréchet differentiable norm, let $T: C \rightarrow C$ be a nonexpansive mapping with a fixed point, and let $\gamma_{n}$ be a sequence of real numbers such that $\gamma_{n} \in[0,1]$ and $\sum_{n=1}^{\infty} \gamma_{n}\left(1-\gamma_{n}\right)=\infty$. Then the sequence $\left\{x_{n}\right\}_{n \in \mathbb{N}}$ generated by Mann's algorithm (1.2) converges weakly to a fixed point of $T$.

However, the convergence of the sequence $\left\{x_{n}\right\}_{n \in \mathbb{N}}$ generated by Mann's algorithm (1.2) is in general not strong (see a counterexample in [18]; see also [19]). Some attempts to modify the Mann iteration method (1.2) so that strong convergence is guaranteed have recently been made. Bauschke and Combettes [4] proposed the following modification of the Mann iteration method for a single nonexpansive mapping $T$ in a Hilbert space $H$ :

$$
\left\{\begin{array}{l}
x_{0}=x \in C, \\
y_{n}=\alpha_{n} x_{n}+\left(1-\alpha_{n}\right) T x_{n}, \\
C_{n}=\left\{z \in C_{n}:\left\|z-y_{n}\right\| \leq\left\|z-x_{n}\right\|\right\}, \\
Q_{n}=\left\{z \in C:\left\langle x_{n}-z, x-x_{n}\right\rangle \geq 0\right\}, \\
x_{n+1}=P_{C_{n} \cap Q_{n}} x,
\end{array}\right.
$$

where $C$ is a closed and convex subset of $H, P_{Q}$ denotes the metric projection from $H$ onto a closed and convex subset $Q$ of $H$. They proved that if the sequence $\left\{\alpha_{n}\right\}_{n \in \mathbb{N}}$ is bounded 
above from one, then the sequence $\left\{x_{n}\right\}_{n \in \mathbb{N}}$ generated by (1.3) converges strongly to $P_{F(T)} x$ as $n \rightarrow \infty$.

Let $E$ be a smooth, strictly convex and reflexive Banach space and let $J$ be a normalized duality mapping of $E$. Let $C$ be a nonempty, closed and convex subset of $E$. The generalized projection $\Pi_{C}$ from $E$ onto $C$ [20] is defined and denoted by

$$
\Pi_{C}(x)=\underset{y \in C}{\arg \min } \phi(y, x),
$$

where $\phi(x, y)=\|x\|^{2}-2\langle x, J y\rangle+\|y\|^{2}$. Let $C$ be a nonempty, closed and convex subset of a smooth Banach space $E$, let $T$ be a mapping from $C$ into itself. A point $p \in C$ is said to be an asymptotic fixed point [21] of $T$ if there exists a sequence $\left\{x_{n}\right\}_{n \in \mathbb{N}}$ in $C$ which converges weakly to $p$ and $\lim _{n \rightarrow \infty}\left\|x_{n}-T x_{n}\right\|=0$. We denote the set of all asymptotic fixed points of $T$ by $\hat{F}(T)$. A point $p \in C$ is called a strong asymptotic fixed point of $T$ if there exists a sequence $\left\{x_{n}\right\}_{n \in \mathbb{N}}$ in $C$ which converges strongly to $p$ and $\lim _{n \rightarrow \infty}\left\|x_{n}-T x_{n}\right\|=0$. We denote the set of all strong asymptotic fixed points of $T$ by $\tilde{F}(T)$.

Following Matsushita and Takahashi [22], a mapping $T: C \rightarrow C$ is said to be relatively nonexpansive if the following conditions are satisfied:

(1) $F(T)$ is nonempty;

(2) $\phi(u, T x) \leq \phi(u, x), \forall u \in F(T), x \in C$;

(3) $\hat{F}(T)=F(T)$.

In 2005, Matsushita and Takahashi [22] proved the following strong convergence theorem for relatively nonexpansive mappings in a Banach space.

Theorem 1.2 Let $E$ be a uniformly smooth and uniformly convex Banach space, let $C$ be a nonempty, closed and convex subset of $E$, let $T$ be a relatively nonexpansive mapping from $C$ into itself, and let $\left\{\alpha_{n}\right\}_{n \in \mathbb{N}}$ be a sequence of real numbers such that $0 \leq \alpha_{n}<1$ and $\limsup _{n \rightarrow \infty} \alpha_{n}<1$. Suppose that $\left\{x_{n}\right\}_{n \in \mathbb{N}}$ is given by

$$
\left\{\begin{array}{l}
x_{0}=x \in C, \\
y_{n}=J^{-1}\left(\alpha_{n} J x_{n}+\left(1-\alpha_{n}\right) J T x_{n}\right), \\
H_{n}=\left\{z \in C_{n}: \phi\left(z, y_{n}\right) \leq \phi\left(z, x_{n}\right)\right\}, \\
W_{n}=\left\{z \in C:\left\langle x_{n}-z, J x-J x_{n}\right\rangle \geq 0\right\}, \\
x_{n+1}=\Pi_{H_{n} \cap W_{n}} x .
\end{array}\right.
$$

If $F(T)$ is nonempty, then $\left\{x_{n}\right\}_{n \in \mathbb{N}}$ converges strongly to $\Pi_{F(T)} x$.

\subsection{Some facts about gradient}

For any convex function $g: E \rightarrow(-\infty,+\infty]$ we denote the domain of $g$ by dom $g=\{x \in$ $E: g(x)<\infty\}$. For any $x \in$ int dom $g$ and any $y \in E$, we denote by $g^{0}(x, y)$ the right-hand derivative of $g$ at $x$ in the direction $y$, that is,

$$
g^{0}(x, y)=\lim _{t \downarrow 0} \frac{g(x+t y)-g(x)}{t} .
$$

The function $g$ is said to be Gâteaux differentiable at $x$ if $\lim _{t \rightarrow 0} \frac{g(x+t y)-g(x)}{t}$ exists for any $y$. In this case, $g^{0}(x, y)$ coincides with $\nabla g(x)$, the value of the gradient $\nabla g$ of $g$ at $x$. The function $g$ is said to be Gâteaux differentiable if it is Gâteaux differentiable everywhere. The 
function $g$ is said to be Fréchet differentiable at $x$ if this limit is attained uniformly in $\|y\|=1$. The function $g$ is Fréchet differentiable at $x \in E$ (see, for example, [23, p.13] or [24, p.508]) if for all $\epsilon>0$, there exists $\delta>0$ such that $\|y-x\| \leq \delta$ implies that

$$
|g(y)-g(x)-\langle y-x, \nabla g(x)\rangle| \leq \epsilon\|y-x\| .
$$

The function $g$ is said to be Fréchet differentiable if it is Fréchet differentiable everywhere. It is well known that if a continuous convex function $g: E \rightarrow \mathbb{R}$ is Gâteaux differentiable, then $\nabla g$ is norm-to-weak* continuous (see, for example, [23, Proposition 1.1.10]). Also, it is known that if $g$ is Fréchet differentiable, then $\nabla g$ is norm-to-norm continuous (see [24, p.508]). The mapping $\nabla g$ is said to be weakly sequentially continuous if $x_{n} \rightarrow x$ as $n \rightarrow \infty$ implies that $\nabla g\left(x_{n}\right) \rightarrow^{*} \nabla g(x)$ as $n \rightarrow \infty$ (for more details, see [23, Theorem 3.2.4] or [24, p.508]). The function $g$ is said to be strongly coercive if

$$
\lim _{\left\|x_{n}\right\| \rightarrow \infty} \frac{g\left(x_{n}\right)}{\left\|x_{n}\right\|}=\infty .
$$

It is also said to be bounded on bounded subsets of $E$ if $g(U)$ is bounded for each bounded subset $U$ of $E$. Finally, $g$ is said to be uniformly Fréchet differentiable on a subset $X$ of $E$ if the limit (1.5) is attained uniformly for all $x \in X$ and $\|y\|=1$.

Let $A: E \rightarrow 2^{E^{*}}$ be a set-valued mapping. We define the domain and range of $A$ by $\operatorname{dom} A=\{x \in E: A x \neq \varnothing\}$ and $\operatorname{ran} A=\bigcup_{x \in E} A x$, respectively. The graph of $A$ is denoted by $G(A)=\left\{\left(x, x^{*}\right) \in E \times E^{*}: x^{*} \in A x\right\}$. The mapping $A \subset E \times E^{*}$ is said to be monotone [25] if $\left\langle x-y, x^{*}-y^{*}\right\rangle \geq 0$ whenever $\left(x, x^{*}\right),\left(y, y^{*}\right) \in A$. It is also said to be maximal monotone [26] if its graph is not contained in the graph of any other monotone operator on $E$. If $A \subset E \times E^{*}$ is maximal monotone, then we can show that the set $A^{-1} 0=\{z \in E: 0 \in A z\}$ is closed and convex. A mapping $A: \operatorname{dom} A \subset E \rightarrow E^{*}$ is called $\gamma$-inverse strongly monotone if there exists a positive real number $\gamma$ such that for all $x, y \in \operatorname{dom} A,\langle x-y, A x-A y\rangle \geq$ $\gamma\|A x-A y\|^{2}$.

\subsection{Some facts about Legendre functions}

Let $E$ be a reflexive Banach space. For any proper, lower semicontinuous and convex function $g: E \rightarrow(-\infty,+\infty]$, the conjugate function $g^{*}$ of $g$ is defined by

$$
g^{*}\left(x^{*}\right)=\sup _{x \in E}\left\{\left\langle x, x^{*}\right\rangle-g(x)\right\}
$$

for all $x^{*} \in E^{*}$. It is well known that $g(x)+g^{*}\left(x^{*}\right) \geq\left\langle x, x^{*}\right\rangle$ for all $\left(x, x^{*}\right) \in E \times E^{*}$. It is also known that $\left(x, x^{*}\right) \in \partial g$ is equivalent to

$$
g(x)+g^{*}\left(x^{*}\right)=\left\langle x, x^{*}\right\rangle
$$

Here, $\partial g$ is the subdifferential of $g[27,28]$. We also know that if $g: E \rightarrow(-\infty,+\infty]$ is a proper, lower semicontinuous and convex function, then $g^{*}: E^{*} \rightarrow(-\infty,+\infty]$ is a proper, weak* lower semicontinuous and convex function; see [15] for more details on convex analysis. 
Let $g: E \rightarrow(-\infty,+\infty]$ be a mapping. The function $g$ is said to be:

(i) essentially smooth, if $\partial g$ is both locally bounded and single-valued on its domain;

(ii) essentially strictly convex, if $(\partial g)^{-1}$ is locally bounded on its domain and $g$ is strictly convex on every convex subset of dom $\partial g$;

(iii) Legendre, if it is both essentially smooth and essentially strictly convex (for more details, we refer to [29, Definition 5.2]).

If $E$ is a reflexive Banach space and $g: E \rightarrow(-\infty,+\infty]$ is a Legendre function, then in view of [30, p.83],

$$
\nabla g^{*}=(\nabla g)^{-1}, \quad \operatorname{ran} \nabla g=\operatorname{dom} g^{*}=\operatorname{int} \operatorname{dom} g^{*} \quad \text { and } \quad \operatorname{ran} \nabla g=\operatorname{int} \operatorname{dom} g
$$

Examples of Legendre functions are given in [29, 31]. One important and interesting Legendre function is $\frac{1}{s}\|\cdot\|^{s}(1<s<\infty)$, where the Banach space $E$ is smooth and strictly convex and, in particular, a Hilbert space.

\subsection{Some facts about Bregman distance}

Let $E$ be a Banach space and let $E^{*}$ be the dual space of $E$. Let $g: E \rightarrow \mathbb{R}$ be a convex and Gâteaux differentiable function. Then the Bregman distance $[32,33]$ corresponding to $g$ is the function $D_{g}: E \times E \rightarrow \mathbb{R}$ defined by

$$
D_{g}(x, y)=g(x)-g(y)-\langle x-y, \nabla g(y)\rangle, \quad \forall x, y \in E .
$$

It is clear that $D_{g}(x, y) \geq 0$ for all $x, y \in E$. In that case when $E$ is a smooth Banach space, setting $g(x)=\|x\|^{2}$ for all $x \in E$, we obtain that $\nabla g(x)=2 J x$ for all $x \in E$ and hence $D_{g}(x, y)=$ $\phi(x, y)$ for all $x, y \in E$.

Let $E$ be a Banach space and let $C$ be a nonempty and convex subset of $E$. Let $g: E \rightarrow \mathbb{R}$ be a convex and Gâteaux differentiable function. Then we know from [34] that for $x \in E$ and $x_{0} \in C, D_{g}\left(x_{0}, x\right)=\min _{y \in C} D_{g}(y, x)$ if and only if

$$
\left\langle y-x_{0}, \nabla g(x)-\nabla g\left(x_{0}\right)\right\rangle \leq 0, \quad \forall y \in C .
$$

Furthermore, if $C$ is a nonempty, closed and convex subset of a reflexive Banach space $E$ and $g: E \rightarrow \mathbb{R}$ is a strongly coercive Bregman function, then for each $x \in E$, there exists a unique $x_{0} \in C$ such that

$$
D_{g}\left(x_{0}, x\right)=\min _{y \in C} D_{g}(y, x)
$$

The Bregman projection proj $_{C}^{g}$ from $E$ onto $C$ is defined by $\operatorname{proj}_{C}^{g}(x)=x_{0}$ for all $x \in E$. It is also well known that proj ${ }_{C}^{g}$ has the following property:

$$
D_{g}\left(y, \operatorname{proj}_{C}^{g} x\right)+D_{g}\left(\operatorname{proj}_{C}^{g} x, x\right) \leq D_{g}(y, x)
$$

for all $y \in C$ and $x \in E$ (see [23] for more details). 


\subsection{Some facts about uniformly convex and totally convex functions}

Let $E$ be a Banach space and let $B_{r}:=\{z \in E:\|z\| \leq r\}$ for all $r>0$. Then a function $g: E \rightarrow$ $\mathbb{R}$ is said to be uniformly convex on bounded subsets of $E$ [35, pp.203, 221] if $\rho_{r}(t)>0$ for all $r, t>0$, where $\rho_{r}:[0,+\infty) \rightarrow[0, \infty]$ is defined by

$$
\rho_{r}(t)=\inf _{x, y \in B_{r},\|x-y\|=t, \alpha \in(0,1)} \frac{\alpha g(x)+(1-\alpha) g(y)-g(\alpha x+(1-\alpha) y)}{\alpha(1-\alpha)}
$$

for all $t \geq 0$. The function $\rho_{r}$ is called the gauge of uniform convexity of $g$. The function $g$ is also said to be uniformly smooth on bounded subsets of $E[35, \mathrm{pp} .207,221] \operatorname{if}_{\lim _{t \downarrow}} \frac{\sigma_{r}(t)}{t}=0$ for all $r>0$, where $\sigma_{r}:[0,+\infty) \rightarrow[0, \infty]$ is defined by

$$
\sigma_{r}(t)=\sup _{x \in B_{r}, y \in S_{E}, \alpha \in(0,1)} \frac{\alpha g(x+(1-\alpha) t y)+(1-\alpha) g(x-\alpha t y)-g(x)}{\alpha(1-\alpha)}
$$

for all $t \geq 0$. The function $g$ is said to be uniformly convex if the function $\delta_{g}:[0,+\infty) \rightarrow$ $[0,+\infty]$, defined by

$$
\delta_{g}(t):=\sup \left\{\frac{1}{2} g(x)+\frac{1}{2} g(y)-g\left(\frac{x+y}{2}\right):\|y-x\|=t\right\},
$$

satisfies that $\lim _{t \downarrow 0} \frac{\sigma_{r}(t)}{t}=0$.

Remark 1.1 Let $E$ be a Banach space, let $r>0$ be a constant and let $g: E \rightarrow \mathbb{R}$ be a convex function which is uniformly convex on bounded subsets. Then

$$
g(\alpha x+(1-\alpha) y) \leq \alpha g(x)+(1-\alpha) g(y)-\alpha(1-\alpha) \rho_{r}(\|x-y\|)
$$

for all $x, y \in B_{r}$ and $\alpha \in(0,1)$, where $\rho_{r}$ is the gauge of uniform convexity of $g$.

Let $g: E \rightarrow(-\infty,+\infty]$ be a convex and Gâteaux differentiable function. Recall that, in view of [23, Section 1.2, p.17] (see also [36]), the function $g$ is called totally convex at a point $x \in \operatorname{int} \operatorname{dom} g$ if its modulus of total convexity at $x$, that is, the function $v_{g}$ : int dom $g \times$ $[0,+\infty) \rightarrow[0,+\infty)$, defined by

$$
v_{g}(x, t):=\inf \left\{D_{g}(y, x): y \in \operatorname{int} \operatorname{dom} g,\|y-x\|=t\right\},
$$

is positive whenever $t>0$. The function $g$ is called totally convex when it is totally convex at every point $x \in$ int dom $g$. Moreover, the function $f$ is called totally convex on bounded subsets of $E$ if $v_{g}(x, t)>0$ for any bounded subset $X$ of $E$ and for any $t>0$, where the modulus of total convexity of the function $g$ on the set $X$ is the function $v_{g}$ : int dom $g \times$ $[0,+\infty) \rightarrow[0,+\infty)$ defined by

$$
v_{g}(X, t):=\inf \left\{v_{g}(x, t): x \in X \cap \operatorname{int} \operatorname{dom} g\right\} .
$$

It is well known that any uniformly convex function is totally convex, but the converse is not true in general (see [23, Section 1.3, p.30]).

It is also well known that $g$ is totally convex on bounded subsets if and only if $g$ is uniformly convex on bounded subsets (see [37, Theorem 2.10, p.9]). 
Examples of totally convex functions can be found, for instance, in [23, 37].

\subsection{Some facts about resolvent}

Let $E$ be a reflexive Banach space with the dual space $E^{*}$ and let $g: E \rightarrow(-\infty,+\infty$ ] be a proper, lower semicontinuous and convex function. Let $A$ be a maximal monotone operator from $E$ to $E^{*}$. For any $r>0$, let the mapping $\operatorname{Res}_{r A}^{g}: E \rightarrow \operatorname{dom} A$ be defined by

$$
\operatorname{Res}_{r A}^{g}=(\nabla g+r A)^{-1} \nabla g
$$

The mapping $\operatorname{Res}_{r A}^{g}$ is called the $g$-resolvent of $A$ (see [38]). It is well known that $A^{-1}(0)=$ $F\left(\operatorname{Res}_{r A}^{g}\right)$ for each $r>0$ (for more details, see, for example, [14]).

Examples and some important properties of such operators are discussed in [39].

\subsection{Some facts about Bregman quasi-nonexpansive mappings}

Let $C$ be a nonempty, closed and convex subset of a reflexive Banach space $E$. Let $g: E \rightarrow$ $(-\infty,+\infty]$ be a proper, lower semicontinuous and convex function. Recall that a mapping $T: C \rightarrow C$ is said to be Bregman quasi-nonexpansive [40] if $F(T) \neq \varnothing$ and

$$
D_{g}(p, T x) \leq D_{g}(p, x), \quad \forall x \in C, p \in F(T) .
$$

A mapping $T: C \rightarrow C$ is said to be Bregman relatively nonexpansive [40] if the following conditions are satisfied:

(1) $F(T)$ is nonempty;

(2) $D_{g}(p, T v) \leq D_{g}(p, v), \forall p \in F(T), v \in C$;

(3) $\hat{F}(T)=F(T)$.

Now, we are in a position to introduce the following new class of Bregman quasinonexpansive type mappings. A mapping $T: C \rightarrow C$ is said to be Bregman weak relatively nonexpansive if the following conditions are satisfied:

(1) $F(T)$ is nonempty;

(2) $D_{g}(p, T v) \leq D_{g}(p, v), \forall p \in F(T), v \in C$;

(3) $\tilde{F}(T)=F(T)$.

It is clear that any Bregman relatively nonexpansive mapping is a Bregman quasinonexpansive mapping. It is also obvious that every Bregman relatively nonexpansive mapping is a Bregman weak relatively nonexpansive mapping, but the converse in not true in general. Indeed, for any mapping $T: C \rightarrow C$, we have $F(T) \subset \tilde{F}(T) \subset \hat{F}(T)$. If $T$ is Bregman relatively nonexpansive, then $F(T)=\tilde{F}(T)=\hat{F}(T)$. Below we show that there exists a Bregman weak relatively nonexpansive mapping which is not a Bregman relatively nonexpansive mapping.

Example 1.1 Let $E=l^{2}$, where

$$
\begin{aligned}
& l^{2}=\left\{\sigma=\left(\sigma_{1}, \sigma_{2}, \ldots, \sigma_{n}, \ldots\right): \sum_{n=1}^{\infty}\left\|\sigma_{n}\right\|^{2}<\infty\right\}, \quad\|\sigma\|=\left(\sum_{n=1}^{\infty}\left\|\sigma_{n}\right\|^{2}\right)^{\frac{1}{2}}, \quad \forall \sigma \in l^{2}, \\
& \langle\sigma, \eta\rangle=\sum_{n=1}^{\infty} \sigma_{n} \eta_{n}, \quad \forall \delta=\left(\sigma_{1}, \sigma_{2}, \ldots, \sigma_{n}, \ldots\right), \eta=\left(\eta_{1}, \eta_{2}, \ldots, \eta_{n}, \ldots\right) \in l^{2} .
\end{aligned}
$$


Let $\left\{x_{n}\right\}_{n \in \mathbb{N} \cup\{0\}} \subset E$ be a sequence defined by

$$
\begin{aligned}
& x_{0}=(1,0,0,0, \ldots), \\
& x_{1}=(1,1,0,0,0, \ldots), \\
& x_{2}=(1,0,1,0,0,0, \ldots), \\
& x_{3}=(1,0,0,1,0,0,0, \ldots), \\
& \ldots, \\
& x_{n}=\left(\sigma_{n, 1}, \sigma_{n, 2}, \ldots, \sigma_{n, k}, \ldots\right), \\
& \ldots,
\end{aligned}
$$

where

$$
\sigma_{n, k}= \begin{cases}1 & \text { if } k=1, n+1, \\ 0 & \text { if } k \neq 1, k \neq n+1\end{cases}
$$

for all $n \in \mathbb{N}$. It is clear that the sequence $\left\{x_{n}\right\}_{n \in \mathbb{N}}$ converges weakly to $x_{0}$. Indeed, for any $\Lambda=\left(\lambda_{1}, \lambda_{2}, \ldots, \lambda_{n}, \ldots\right) \in l^{2}=\left(l^{2}\right)^{*}$, we have

$$
\Lambda\left(x_{n}-x_{0}\right)=\left\langle x_{n}-x_{0}, \Lambda\right\rangle=\sum_{k=2}^{\infty} \lambda_{k} \sigma_{n, k} \rightarrow 0
$$

as $n \rightarrow \infty$. It is also obvious that $\left\|x_{n}-x_{m}\right\|=\sqrt{2}$ for any $n \neq m$ with $n, m$ sufficiently large. Thus, $\left\{x_{n}\right\}_{n \in \mathbb{N}}$ is not a Cauchy sequence. Let $k$ be an even number in $\mathbb{N}$ and let $g: E \rightarrow \mathbb{R}$ be defined by

$$
g(x)=\frac{1}{k}\|x\|^{k}, \quad x \in E .
$$

It is easy to show that $\nabla g(x)=J_{k}(x)$ for all $x \in E$, where

$$
J_{k}(x)=\left\{x^{*} \in E^{*}:\left\langle x, x^{*}\right\rangle=\|x\|\left\|x^{*}\right\|,\left\|x^{*}\right\|=\|x\|^{k-1}\right\} .
$$

It is also obvious that

$$
J_{k}(\lambda x)=\lambda^{k-1} J_{k}(x), \quad \forall x \in E, \lambda \in \mathbb{R} .
$$

Now, we define a mapping $T: E \rightarrow E$ by

$$
T(x)= \begin{cases}\frac{n}{n+1} x & \text { if } x=x_{n} \\ -x & \text { if } x \neq x_{n}\end{cases}
$$

It is clear that $F(T)=\{0\}$ and for any $n \in \mathbb{N}$,

$$
\begin{aligned}
D_{g}\left(0, T x_{n}\right) & =g(0)-g\left(T x_{n}\right)-\left\langle 0-T x_{n}, \nabla g\left(T x_{n}\right)\right\rangle \\
& =-\frac{n^{k}}{(n+1)^{k}} g\left(x_{n}\right)+\frac{n^{k}}{(n+1)^{k}}\left\langle x_{n}, \nabla g\left(x_{n}\right)\right\rangle
\end{aligned}
$$




$$
\begin{aligned}
& =\frac{n^{k}}{(n+1)^{k}}\left[-g\left(x_{n}\right)+\left\langle x, \nabla g\left(x_{n}\right)\right\rangle\right] \\
& =\frac{n^{k}}{(n+1)^{k}} D_{g}\left(0, x_{n}\right) \\
& \leq D_{g}\left(0, x_{n}\right) .
\end{aligned}
$$

If $x \neq x_{n}$, then we have

$$
\begin{aligned}
D_{g}(0, T x) & =g(0)-g(T x)-\langle 0-T x, \nabla g(T x)\rangle \\
& =-g(x)-\langle x,-\nabla g(x)\rangle \\
& =-g(x)-\langle-x, \nabla g(x)\rangle \\
& =D_{g}(0, x) .
\end{aligned}
$$

Therefore, $T$ is a Bregman quasi-nonexpansive mapping. Next, we claim that $T$ is a Bregman weak relatively nonexpansive mapping. Indeed, for any sequence $\left\{z_{n}\right\}_{n \in \mathbb{N}} \subset E$ such that $z_{n} \rightarrow z_{0}$ and $\left\|z_{n}-T z_{n}\right\| \rightarrow 0$ as $n \rightarrow \infty$, since $\left\{x_{n}\right\}_{n \in \mathbb{N}}$ is not a Cauchy sequence, there exists a sufficiently large number $N \in \mathbb{N}$ such that $z_{n} \neq x_{m}$ for any $n, m>N$. If we suppose that there exists $m \leq N$ such that $z_{n}=x_{m}$ for infinitely many $n \in \mathbb{N}$, then a subsequence $\left\{x_{n_{i}}\right\}_{i \in \mathbb{N}}$ would satisfy $z_{n_{i}}=x_{m}$, so $z_{0}=\lim _{i \rightarrow \infty} z_{n_{i}}=x_{m}$ and $z_{0}=\lim _{i \rightarrow \infty} T z_{n_{i}}=$ $T x_{m}=\frac{m}{m+1} x_{m}$, which is impossible. This implies that $T z_{n}=-z_{n}$ for all $n>N$. It follows from $\left\|z_{n}-T z_{n}\right\| \rightarrow 0$ that $2 z_{n} \rightarrow 0$ and hence $z_{n} \rightarrow z_{0}=0$. Since $z_{0} \in F(T)$, we conclude that $T$ is a Bregman weak relatively nonexpansive mapping.

Finally, we show that $T$ is not Bregman relatively nonexpansive. In fact, though $x_{n} \rightarrow x_{0}$ and

$$
\left\|x_{n}-T x_{n}\right\|=\left\|x_{n}-\frac{n}{n+1} x_{n}\right\|=\frac{1}{n+1}\left\|x_{n}\right\| \rightarrow 0
$$

as $n \rightarrow \infty$, but $x_{0} \notin F(T)$. Thus we have $\hat{F}(T) \neq F(T)$.

Let us give an example of a Bregman quasi-nonexpansive mapping which is neither a Bregman relatively nonexpansive mapping nor a Bregman weak relatively nonexpansive mapping (see also [41]).

Example 1.2 Let $E$ be a smooth Banach space, let $k$ be an even number in $\mathbb{N}$ and let $g$ : $E \rightarrow \mathbb{R}$ be defined by

$$
g(x)=\frac{1}{k}\|x\|^{k}, \quad x \in E .
$$

Let $x_{0} \neq 0$ be any element of $E$. We define a mapping $T: E \rightarrow E$ by

$$
T(x)= \begin{cases}\left(\frac{1}{2}+\frac{1}{2^{n+1}}\right) x_{0} & \text { if } x=\left(\frac{1}{2}+\frac{1}{2^{n}}\right) x_{0} \\ -x & \text { if } x \neq\left(\frac{1}{2}+\frac{1}{2^{n}}\right) x_{0}\end{cases}
$$


for all $n \geq 0$. It could easily be seen that $T$ is neither a Bregman weak relatively nonexpansive mapping nor a Bregman relatively nonexpansive mapping. To this end, we set

$$
x_{n}=\left(\frac{1}{2}+\frac{1}{2^{n}}\right) x_{0}, \quad \forall n \in \mathbb{N} .
$$

Though $x_{n} \rightarrow \frac{1}{2} x_{0}\left(x_{n} \rightarrow \frac{1}{2} x_{0}\right)$ as $n \rightarrow \infty$ and

$$
\left\|x_{n}-T x_{n}\right\|=\left\|\left(\frac{1}{2}+\frac{1}{2^{n}}\right) x_{0}-\left(\frac{1}{2}+\frac{1}{2^{n+1}}\right) x_{0}\right\|=\frac{1}{2^{n-1}}\left\|x_{0}\right\| \rightarrow 0
$$

as $n \rightarrow \infty$, but $\frac{1}{2} x_{0} \notin F(T)$. Therefore, $\hat{F}(T) \neq F(T)$ and $\tilde{F}(T) \neq F(T)$.

In [42], Bauschke and Combettes introduced an iterative method to construct the Bregman projection of a point onto a countable intersection of closed and convex sets in reflexive Banach spaces. They proved a strong convergence theorem of the sequence produced by their method; for more detail, see [42, Theorem 4.7].

In [40], Reich and Sabach introduced a proximal method for finding common zeros of finitely many maximal monotone operators in a reflexive Banach space. More precisely, they proved the following strong convergence theorem.

Theorem 1.3 Let $E$ be a reflexive Banach space and let $A_{i}: E \rightarrow 2^{E^{*}}, i=1,2, \ldots, N$, be $N$ maximal monotone operators such that $Z:=\bigcap_{i=1}^{N} A_{i}^{-1}\left(0^{*}\right) \neq \varnothing$. Let $g: E \rightarrow \mathbb{R}$ be a Legendre function that is bounded, uniformly Fréchet differentiable and totally convex on bounded subsets of E. Let $\left\{x_{n}\right\}_{n \in \mathbb{N}}$ be a sequence defined by the following iterative algorithm:

$$
\left\{\begin{array}{l}
x_{0} \in E \quad \text { chosen arbitrarily, } \\
y_{n}^{i}=\operatorname{Res}_{\lambda_{n}^{i} A_{i}}^{g}\left(x_{n}+e_{n}^{i}\right), \\
C_{n}^{i}=\left\{z \in E: D_{g}\left(z, y_{n}^{i}\right) \leq D_{g}\left(z, x_{n}+e_{n}^{i}\right)\right\}, \\
C_{n}:=\bigcap_{i=1}^{N} C_{n}^{i}, \\
Q_{n}=\left\{z \in E:\left\langle\nabla g\left(x_{0}\right)-\nabla g\left(x_{n}\right), z-x_{n}\right\rangle \leq 0\right\}, \\
x_{n+1}=\operatorname{proj}_{C_{n} \cap Q_{n}}^{g} x_{0} \text { and } n \in \mathbb{N} \cup\{0\} .
\end{array}\right.
$$

If, for each $i=1,2, \ldots, N$, $\liminf _{n \rightarrow \infty} \lambda_{n}^{i}>0$ and the sequences of errors $\left\{e_{n}^{i}\right\}_{n \in \mathbb{N}} \subset E$ satisfy $\liminf _{n \rightarrow \infty} e_{n}^{i}=0$, then each such sequence $\left\{x_{n}\right\}_{n \in \mathbb{N}}$ converges strongly to $\operatorname{proj}_{Z}^{g}\left(x_{0}\right)$ as $n \rightarrow \infty$.

Let $C$ be a nonempty, closed and convex subset of a reflexive Banach space $E$. Let $g: E \rightarrow$ $(-\infty,+\infty]$ be a proper, lower semicontinuous and convex function. Recall that a mapping $T: C \rightarrow C$ is said to be Bregman firmly nonexpansive (for short, BFNE) if

$$
D_{g}(T x, T y)+D_{g}(T y, T x)+D_{g}(T x, x)+D_{g}(T y, y) \leq D_{g}(T x, y)+D_{g}(T y, x)
$$

for all $x, y \in C$. The mapping $T$ is called quasi-Bregman firmly nonexpansive (for short, QBFNE) [43], if $F(T) \neq \varnothing$ and

$$
D_{g}(p, T x)+D_{g}(T x, x) \leq D_{g}(p, x)
$$


for all $x \in C$ and $p \in F(T)$. It is clear that any quasi-Bregman firmly nonexpansive mapping is Bregman quasi-nonexpansive. For more information on Bregman firmly nonexpansive mappings, we refer the readers to [38, 44]. In [44], Reich and Sabach proved that for any BFNE operator $T, \hat{F}(T)=F(T)$.

In [43], Reich and Sabach introduced a Mann-type process to approximate fixed points of quasi-Bregman firmly nonexpansive mappings defined on a nonempty, closed and convex subset $C$ of a reflexive Banach space $E$. More precisely, they proved the following theorem.

Theorem 1.4 Let $E$ be a reflexive Banach space and let $T_{i}: E \rightarrow E, i=1,2, \ldots, N$, be $N$ QBFNE operators which satisfy $F\left(T_{i}\right)=\hat{F}\left(T_{i}\right)$ for each $1 \leq i \leq N$ and $F:=\bigcap_{i=1}^{N} F\left(T_{i}\right) \neq \varnothing$. Let $g: E \rightarrow \mathbb{R}$ be a Legendre function that is bounded, uniformly Fréchet differentiable and totally convex on bounded subsets of $E$. Let $\left\{x_{n}\right\}_{n \in \mathbb{N}}$ be a sequence defined by the following iterative algorithm:

$$
\left\{\begin{array}{l}
x_{0} \in E \quad \text { chosen arbitrarily, } \\
Q_{0}^{i}=E, \quad i=1,2, \ldots, N, \\
y_{n}^{i}=T_{i}\left(x_{n}+e_{n}^{i}\right), \\
Q_{n+1}^{i}=\left\{z \in Q_{n}^{i}:\left\langle\nabla g\left(x_{n}+e_{n}^{i}\right)-\nabla g\left(y_{n}^{i}\right), z-y_{n}^{i}\right\rangle \leq 0\right\}, \\
Q_{n+1}:=\bigcap_{i=1}^{N} Q_{n+1}^{i}, \\
x_{n+1}=\operatorname{proj}_{Q_{n+1}}^{g} x_{0} \text { and } n \in \mathbb{N} \cup\{0\} .
\end{array}\right.
$$

If, for each $i=1,2, \ldots, N$, the sequences of errors $\left\{e_{n}^{i}\right\}_{n \in \mathbb{N}} \subset E$ satisfy $\liminf _{n \rightarrow \infty} e_{n}^{i}=0$, then each such sequence $\left\{x_{n}\right\}_{n \in \mathbb{N}}$ converges strongly to $\operatorname{proj}_{F}^{g}\left(x_{0}\right)$ as $n \rightarrow \infty$.

Let $E$ be a reflexive Banach space and let $g: E \rightarrow \mathbb{R}$ be a convex and Gâteaux differentiable function. Let $C$ be a nonempty, closed and convex subset of $E$. Recall that a mapping $T: C \rightarrow C$ is said to be (quasi-)Bregman strongly firmly nonexpansive (for short, BSNE) with respect to a nonempty $\hat{F}(T)$ if $F(T) \neq \varnothing$ and

$$
D_{g}(p, T x) \leq D_{g}(p, x)
$$

for all $x \in C$ and $p \in \hat{F}(T)$, and if whenever $\left\{x_{n}\right\}_{n \in \mathbb{N}} \subset C$ is bounded and $p \in F(T)$, then we have

$$
\lim _{n \rightarrow \infty}\left(D_{g}\left(p, x_{n}\right)-D_{g}\left(p, T x_{n}\right)\right)=0 \Longrightarrow \lim _{n \rightarrow \infty} D_{g}\left(T x_{n}, x_{n}\right)=0 .
$$

The class of (quasi-)Bregman strongly nonexpansive mappings was first introduced in [21, 45] (for more details, see also [46]). We know that the notion of a strongly nonexpansive operator (with respect to the norm) was first introduced and studied in [47, 48].

In [46], Reich and Sabach introduced iterative algorithms for finding common fixed points of finitely many Bregman strongly nonexpansive operators in a reflexive Banach space. They established the following strong convergence theorem in a reflexive Banach space.

Theorem 1.5 Let $E$ be a reflexive Banach space and let $T_{i}: E \rightarrow E, i=1,2, \ldots, N$, be $N$ BSNE operators which satisfy $F\left(T_{i}\right)=\hat{F}\left(T_{i}\right)$ for each $1 \leq i \leq N$ and $F:=\bigcap_{i=1}^{N} F\left(T_{i}\right) \neq \varnothing$. 
Let $g: E \rightarrow \mathbb{R}$ be a Legendre function that is bounded, uniformly Fréchet differentiable and totally convex on bounded subsets of E. Let $\left\{x_{n}\right\}_{n \in \mathbb{N}}$ be a sequence defined by the following iterative algorithm:

$$
\left\{\begin{array}{l}
x_{0} \in E \quad \text { chosen arbitrarily, } \\
y_{n}^{i}=T_{i}\left(x_{n}+e_{n}^{i}\right), \\
C_{n}^{i}=\left\{z \in E: D_{g}\left(z, y_{n}^{i}\right) \leq D_{g}\left(z, x_{n}+e_{n}^{i}\right)\right\}, \\
C_{n}:=\bigcap_{i=1}^{N} C_{n}^{i}, \\
Q_{n}=\left\{z \in E:\left\langle\nabla g\left(x_{0}\right)-\nabla g\left(x_{n}\right), z-x_{n}\right\rangle \leq 0\right\}, \\
x_{n+1}=\operatorname{proj}_{C_{n} \cap Q_{n}}^{g} x_{0} \text { and } n \in \mathbb{N} \cup\{0\} .
\end{array}\right.
$$

If, for each $i=1,2, \ldots, N$, the sequences of errors $\left\{e_{n}^{i}\right\}_{n \in \mathbb{N}} \subset E$ satisfy $\liminf _{n \rightarrow \infty} e_{n}^{i}=0$, then each such sequence $\left\{x_{n}\right\}_{n \in \mathbb{N}}$ converges strongly to $\operatorname{proj}_{F}^{g}\left(x_{0}\right)$ as $n \rightarrow \infty$.

But it is worth mentioning that, in all the above results for Bregman nonexpansive-type mappings, the assumption $\hat{F}(T)=F(T)$ is imposed on the map $T$.

Remark 1.2 Though the iteration processes (1.10) and (1.12), as introduced by the authors mentioned above, worked, it is easy to see that these processes seem cumbersome and complicated in the sense that at each stage of iteration, two different sets $C_{n}$ and $Q_{n}$ are computed and the next iterate taken as the Bregman projection of $x_{0}$ on the intersection of $C_{n}$ and $Q_{n}$. This seems difficult to do in application. It is important to state clearly that the iteration process (1.11) involves computation of only one set $Q_{n}$ at each stage of iteration. In [49], Sabach proposed an excellent modification of algorithm (1.10) for finding common zeros of finitely many maximal monotone operators in reflexive Banach spaces.

Our concern now is the following:

Is it possible to obtain strong convergence of modified Mann-type schemes (1.10)(1.12) to a fixed point of a Bregman quasi-nonexpansive type mapping $T$ without imposing the assumption $\hat{F}(T)=F(T)$ on $T$ ?

In this paper, using Bregman functions, we introduce new hybrid iterative algorithms for finding common fixed points of an infinite family of Bregman weak relatively nonexpansive mappings in Banach spaces. We prove strong convergence theorems for the sequences produced by the methods. Furthermore, we apply our method to prove strong convergence theorems of iterative algorithms for finding common fixed points of finitely many Bregman weak relatively nonexpansive mappings in reflexive Banach spaces. These algorithms take into account possible computational errors. We also apply our main results to solve equilibrium problems in reflexive Banach spaces. Finally, we study hybrid iterative schemes for finding common solutions of an equilibrium problem, fixed points of an infinite family of Bregman weak relatively nonexpansive mappings and null spaces of a $\gamma$-inverse strongly monotone mapping in 2-uniformly convex Banach spaces. Some application of our results to the solution of equations of Hammerstein type is presented. No assumption $\hat{F}(T)=F(T)$ is imposed on the mapping $T$. Consequently, the above concern is answered in the affirmative in reflexive Banach space setting. Our results improve 
and generalize many known results in the current literature; see, for example, $[4,7,8,11$, $22,40,42-44,46,50-52]$.

\section{Preliminaries}

In this section, we begin by recalling some preliminaries and lemmas which will be used in the sequel.

The following definition is slightly different from that in Butnariu and Iusem [23].

Definition 2.1 [24] Let $E$ be a Banach space. The function $g: E \rightarrow \mathbb{R}$ is said to be a Bregman function if the following conditions are satisfied:

(1) $g$ is continuous, strictly convex and Gâteaux differentiable;

(2) the set $\left\{y \in E: D_{g}(x, y) \leq r\right\}$ is bounded for all $x \in E$ and $r>0$.

The following lemma follows from Butnariu and Iusem [23] and Zălinscu [35].

Lemma 2.1 Let $E$ be a reflexive Banach space and let $g: E \rightarrow \mathbb{R}$ be a strongly coercive Bregman function. Then

(1) $\nabla g: E \rightarrow E^{*}$ is one-to-one, onto and norm-to-weak ${ }^{*}$ continuous;

(2) $\langle x-y, \nabla g(x)-\nabla g(y)\rangle=0$ if and only if $x=y$;

(3) $\left\{x \in E: D_{g}(x, y) \leq r\right\}$ is bounded for all $y \in E$ and $r>0$;

(4) $\operatorname{dom} g^{*}=E^{*}, g^{*}$ is Gâteaux differentiable and $\nabla g^{*}=(\nabla g)^{-1}$.

Now, we are ready to prove the following key lemma.

Lemma 2.2 Let $E$ be a Banach space, let $r>0$ be a constant and let $g: E \rightarrow \mathbb{R}$ be a convex function which is uniformly convex on bounded subsets of $E$. Then

$$
g\left(\sum_{k=0}^{n} \alpha_{k} x_{k}\right) \leq \sum_{k=0}^{n} \alpha_{k} g\left(x_{k}\right)-\alpha_{i} \alpha_{j} \rho_{r}\left(\left\|x_{i}-x_{j}\right\|\right)
$$

for all $i, j \in\{0,1,2, \ldots, n\}, x_{k} \in B_{r}, \alpha_{k} \in(0,1)$ and $k=0,1,2, \ldots, n$ with $\sum_{k=0}^{n} \alpha_{k}=1$, where $\rho_{r}$ is the gauge of uniform convexity of $g$.

Proof Without loss of generality, we may assume that $i=0$ and $j=1$. By induction on $n$, for $n=1$, in view of Remark 1.1 we get the desired result. Now suppose that it is true for $n=k$, i.e.,

$$
g\left(\sum_{m=0}^{k} \alpha_{m} x_{m}\right) \leq \sum_{m=0}^{k} \alpha_{m} g\left(x_{m}\right)-\alpha_{0} \alpha_{1} \rho_{r}\left(\left\|x_{0}-x_{1}\right\|\right) .
$$

Now, we prove that the conclusion holds for $n=k+1$. Put $x=\frac{\sum_{m=0}^{k} \alpha_{m} x_{m}}{1-\alpha_{k+1}}$ and observe that $x \in B_{r}$. Since $g$ is convex, given assumption, we conclude that

$$
\begin{aligned}
g\left(\sum_{m=0}^{k+1} \alpha_{m} x_{m}\right) & =g\left(\left(1-\alpha_{k+1}\right) \sum_{m=0}^{k} \frac{\alpha_{m} x_{m}}{1-\alpha_{k+1}}+\alpha_{k+1} x_{k+1}\right) \\
& \leq\left(1-\alpha_{k+1}\right) g\left(\sum_{m=0}^{k} \frac{\alpha_{m} x_{m}}{1-\alpha_{k+1}}\right)+\alpha_{k+1} g\left(x_{k+1}\right)
\end{aligned}
$$




$$
\begin{aligned}
& \leq \sum_{m=0}^{k} \alpha_{m} g\left(x_{m}\right)-\alpha_{0} \alpha_{1} \rho_{r}\left(\left\|x_{0}-x_{1}\right\|\right)+\alpha_{k+1} g\left(x_{k+1}\right) \\
& =\sum_{m=0}^{k+1} \alpha_{m} g\left(x_{m}\right)-\alpha_{0} \alpha_{1} \rho_{r}\left(\left\|x_{0}-x_{1}\right\|\right) .
\end{aligned}
$$

This completes the proof.

Lemma 2.3 Let $E$ be a Banach space, let $r>0$ be a constant and let $g: E \rightarrow \mathbb{R}$ be a continuous and convex function which is uniformly convex on bounded subsets of E. Then

$$
g\left(\sum_{k=0}^{\infty} \alpha_{k} x_{k}\right) \leq \sum_{k=0}^{\infty} \alpha_{k} g\left(x_{k}\right)-\alpha_{i} \alpha_{j} \rho_{r}\left(\left\|x_{i}-x_{j}\right\|\right)
$$

for all $i, j \in \mathbb{N} \cup\{0\}, x_{k} \in B_{r}, \alpha_{k} \in(0,1)$ and $k \in \mathbb{N} \cup\{0\}$ with $\sum_{k=0}^{\infty} \alpha_{k}=1$, where $\rho_{r}$ is the gauge of uniform convexity of $g$.

Proof Let $i, j \in \mathbb{N} \cup\{0\}$ and $k>i, j$. Put $v_{k}=\frac{\alpha_{0} x_{0}}{\sum_{m=0}^{k} \alpha_{m}}+\frac{\alpha_{1} x_{1}}{\sum_{m=0}^{k} \alpha_{m}}+\cdots+\frac{\alpha_{k} x_{k}}{\sum_{m=0}^{k} \alpha_{m}}$ and observe that $v_{k} \in B_{r}$ for all $k \in \mathbb{N}$. In view of Lemma 2.2 , we obtain that

$$
\begin{aligned}
g\left(v_{k}\right) & =g\left(\frac{\alpha_{0} x_{0}}{\sum_{m=0}^{k} \alpha_{m}}+\frac{\alpha_{1} x_{1}}{\sum_{m=0}^{k} \alpha_{m}}+\cdots+\frac{\alpha_{k} x_{k}}{\sum_{m=0}^{k} \alpha_{m}}\right) \\
& \leq \frac{1}{\sum_{m=0}^{k} \alpha_{m}} \sum_{m=0}^{k} \alpha_{m} g\left(x_{m}\right)-\alpha_{i} \alpha_{j} \rho_{r}\left(\left\|x_{i}-x_{j}\right\|\right) .
\end{aligned}
$$

Since $g$ is continuous and $v_{k} \rightarrow \sum_{m=0}^{\infty} \alpha_{m} x_{m}$ as $k \rightarrow \infty$, we have

$$
\lim _{k \rightarrow \infty} g\left(v_{k}\right)=g\left(\sum_{m=0}^{\infty} \alpha_{m} x_{m}\right)
$$

Letting $k \rightarrow \infty$ in $(2.1)$, we conclude that

$$
g\left(\sum_{m=0}^{\infty} \alpha_{m} x_{m}\right) \leq \sum_{m=0}^{\infty} \alpha_{m} g\left(x_{m}\right)-\alpha_{i} \alpha_{j} \rho_{r}\left(\left\|x_{i}-x_{j}\right\|\right)
$$

which completes the proof.

We know the following two results; see [35, Proposition 3.6.4].

Theorem 2.1 Let $E$ be a reflexive Banach space and let $g: E \rightarrow \mathbb{R}$ be a convex function which is bounded on bounded subsets of E. Then the following assertions are equivalent:

(1) $g$ is strongly coercive and uniformly convex on bounded subsets of $E$;

(2) $\operatorname{dom} g^{*}=E^{*}, g^{*}$ is bounded on bounded subsets and uniformly smooth on bounded subsets of $E^{*}$;

(3) $\operatorname{dom} g^{*}=E^{*}, g^{*}$ is Fréchet differentiable and $\nabla g^{*}$ is uniformly norm-to-norm continuous on bounded subsets of $E^{*}$. 
Theorem 2.2 Let $E$ be a reflexive Banach space and let $g: E \rightarrow \mathbb{R}$ be a continuous convex function which is strongly coercive. Then the following assertions are equivalent:

(1) $g$ is bounded on bounded subsets and uniformly smooth on bounded subsets of $E$;

(2) $g^{*}$ is Fréchet differentiable and $\nabla g^{*}$ is uniformly norm-to-norm continuous on bounded subsets of $E^{*}$;

(3) $\operatorname{dom} g^{*}=E^{*}, g^{*}$ is strongly coercive and uniformly convex on bounded subsets of $E^{*}$.

Let $E$ be a Banach space and let $g: E \rightarrow \mathbb{R}$ be a convex and Gâteaux differentiable function. Then the Bregman distance [32,33] satisfies the three point identity that is

$$
D_{g}(x, z)=D_{g}(x, y)+D_{g}(y, z)+\langle x-y, \nabla g(y)-\nabla g(z)\rangle, \quad \forall x, y, z \in E .
$$

In particular, it can be easily seen that

$$
D_{g}(x, y)=-D_{g}(y, x)+\langle y-x, \nabla g(y)-\nabla g(x)\rangle, \quad \forall x, y \in E .
$$

Indeed, by letting $z=x$ in (2.2) and taking into account that $D_{g}(x, x)=0$, we get the desired result.

Lemma 2.4 Let E be a Banach space and let $g: E \rightarrow \mathbb{R}$ be a Gâteaux differentiable function which is uniformly convex on bounded subsets of E. Let $\left\{x_{n}\right\}_{n \in \mathbb{N}}$ and $\left\{y_{n}\right\}_{n \in \mathbb{N}}$ be bounded sequences in $E$. Then the following assertions are equivalent:

(1) $\lim _{n \rightarrow \infty} D_{g}\left(x_{n}, y_{n}\right)=0$;

(2) $\lim _{n \rightarrow \infty}\left\|x_{n}-y_{n}\right\|=0$.

Proof The implication (1) $\Longrightarrow(2)$ was proved in [23] (see also [24]). For the converse implication, we assume that $\lim _{n \rightarrow \infty}\left\|x_{n}-y_{n}\right\|=0$. Then, in view of (2.3), we have

$$
\begin{aligned}
D_{g}\left(x_{n}, y_{n}\right) & =-D_{g}\left(y_{n}, x_{n}\right)+\left\langle x_{n}-y_{n}, \nabla g\left(x_{n}\right)-\nabla g\left(y_{n}\right)\right\rangle \\
& \leq\left\|x_{n}-y_{n}\right\|\left\|\nabla g\left(x_{n}\right)-\nabla g\left(y_{n}\right)\right\|, \quad \forall n \in \mathbb{N} .
\end{aligned}
$$

The function $g$ is bounded on bounded subsets of $E$ and therefore $\nabla g$ is also bounded on bounded subsets of $E^{*}$ (see, for example, [23, Proposition 1.1.11] for more details). This, together with (2.3)-(2.4), implies that $\lim _{n \rightarrow \infty} D_{g}\left(x_{n}, y_{n}\right)=0$, which completes the proof.

The following result was first proved in [37] (see also [24]).

Lemma 2.5 Let $E$ be a reflexive Banach space, let $g: E \rightarrow \mathbb{R}$ be a strongly coercive Bregman function and let $V$ be the function defined by

$$
V\left(x, x^{*}\right)=g(x)-\left\langle x, x^{*}\right\rangle+g^{*}\left(x^{*}\right), \quad x \in E, x^{*} \in E^{*} .
$$

Then the following assertions hold:

(1) $D_{g}\left(x, \nabla g^{*}\left(x^{*}\right)\right)=V\left(x, x^{*}\right)$ for all $x \in E$ and $x^{*} \in E^{*}$.

(2) $V\left(x, x^{*}\right)+\left\langle\nabla g^{*}\left(x^{*}\right)-x, y^{*}\right\rangle \leq V\left(x, x^{*}+y^{*}\right)$ for all $x \in E$ and $x^{*}, y^{*} \in E^{*}$. 
Corollary 2.1 [35] Let E be a Banach space, let $g: E \rightarrow(-\infty, \infty$ ] be a proper, lower semicontinuous and convex function and let $p, q \in \mathbb{R}$ with $1 \leq p \leq 2 \leq q$ and $p^{-1}+q^{-1}=1$. Then the following statements are equivalent.

(1) There exists $c_{1}>0$ such that $g$ is $\rho$-convex with $\rho(t):=\frac{c_{1}}{q} t^{q}$ for all $t \geq 0$.

(2) There exists $c_{2}>0$ such that for all $\left(x, x^{*}\right),\left(y, y^{*}\right) \in G(\partial g) ;\left\|x^{*}-y^{*}\right\| \geq \frac{2 c_{2}}{q}\|x-y\|^{q-1}$.

\section{Strong convergence theorems without computational errors}

In this section, we prove strong convergence theorems without computational errors in a reflexive Banach space. We start with the following simple lemma whose proof will be omitted since it can be proved by a similar argument as that in [44, Lemma 15.5].

Lemma 3.1 Let $E$ be a reflexive Banach space and let $g: E \rightarrow \mathbb{R}$ be a convex, continuous, strongly coercive and Gâteaux differentiable function which is bounded on bounded subsets and uniformly convex on bounded subsets of E. Let $C$ be a nonempty, closed and convex subset of E. Let $T: C \rightarrow C$ be a Bregman weak relatively nonexpansive mapping. Then $F(T)$ is closed and convex.

Using ideas in [22], we can prove the following result.

Theorem 3.1 Let $E$ be a reflexive Banach space and let $g: E \rightarrow \mathbb{R}$ be a strongly coercive Bregman function which is bounded on bounded subsets and uniformly convex and uniformly smooth on bounded subsets of E. Let $C$ be a nonempty, closed and convex subset of $E$ and let $\left\{T_{j}\right\}_{j \in \mathbb{N}}$ be an infinite family of Bregman weak relatively nonexpansive mappings from $C$ into itself such that $F:=\bigcap_{j=1}^{\infty} F\left(T_{j}\right) \neq \varnothing$. Suppose in addition that $T_{j}^{0}=T_{0}=I$ for all $j \in \mathbb{N}$, where I is the identity mapping on $E$. Let $\left\{x_{n}\right\}_{n \in \mathbb{N}}$ be a sequence generated by

$$
\left\{\begin{array}{l}
x_{0}=x \in C \quad \text { chosen arbitrarily, } \\
C_{0}=C, \\
z_{n}=\nabla g^{*}\left[\alpha_{n, 0} \nabla g\left(x_{n}\right)+\sum_{j=1}^{\infty} \alpha_{n, j} \nabla g\left(T_{j} x_{n}\right)\right], \\
y_{n}=\nabla g^{*}\left[\beta_{n} \nabla g\left(x_{n}\right)+\left(1-\beta_{n}\right) \nabla g\left(z_{n}\right)\right], \\
C_{n+1}=\left\{z \in C_{n}: D_{g}\left(z, y_{n}\right) \leq D_{g}\left(z, x_{n}\right)\right\}, \\
x_{n+1}=\operatorname{proj}_{C_{n+1}}^{g} x \quad \text { and } \quad n \in \mathbb{N} \cup\{0\},
\end{array}\right.
$$

where $\nabla g$ is the right-hand derivative of $g$. Let $\left\{\alpha_{n, j}: j, n \in \mathbb{N} \cup\{0\}\right\}$ and $\left\{\beta_{n}\right\}_{n \in \mathbb{N} \cup\{0\}}$ be sequences in $[0,1)$ satisfying the following control conditions:

(1) $\sum_{j=0}^{\infty} \alpha_{n, j}=1, \forall n \in \mathbb{N} \cup\{0\}$;

(2) There exists $i \in \mathbb{N}$ such that $\liminf _{n \rightarrow \infty} \alpha_{n, i} \alpha_{n, j}>0, \forall j \in \mathbb{N} \cup\{0\}$;

(3) $0 \leq \beta_{n}<1$ for all $n \in \mathbb{N} \cup\{0\}$ and $\lim _{\sup _{n \rightarrow \infty}} \beta_{n}<1$.

Then the sequence $\left\{x_{n}\right\}_{n \in \mathbb{N}}$ defined in (3.1) converges strongly to $\operatorname{proj}_{F}^{g} x$ as $n \rightarrow \infty$.

Proof We divide the proof into several steps.

Step 1. We show that $C_{n}$ is closed and convex for each $n \in \mathbb{N} \cup\{0\}$.

It is clear that $C_{0}=C$ is closed and convex. Let $C_{m}$ be closed and convex for some $m \in \mathbb{N}$. For $z \in C_{m}$, we see that

$$
D_{g}\left(z, y_{m}\right) \leq D_{g}\left(z, x_{m}\right)
$$


is equivalent to

$$
\left\langle z, \nabla g\left(x_{m}\right)-\nabla g\left(y_{m}\right)\right\rangle \leq g\left(y_{m}\right)-g\left(x_{m}\right)+\left\langle x_{m}, \nabla g\left(x_{m}\right)\right\rangle-\left\langle y_{m}, \nabla g\left(y_{m}\right)\right\rangle .
$$

An easy argument shows that $C_{m+1}$ is closed and convex. Hence $C_{n}$ is closed and convex for each $n \in \mathbb{N} \cup\{0\}$.

Step 2. We claim that $F \subset C_{n}$ for all $n \in \mathbb{N} \cup\{0\}$.

It is obvious that $F \subset C_{0}=C$. Assume now that $F \subset C_{m}$ for some $m \in \mathbb{N}$. Employing Lemma 2.5, for any $w \in F \subset C_{m}$, we obtain

$$
\begin{aligned}
D_{g}\left(w, z_{m}\right)= & D_{g}\left(w, \nabla g^{*}\left[\alpha_{m, 0} \nabla g\left(x_{m}\right)+\sum_{j=1}^{\infty} \alpha_{m, j} \nabla g\left(T_{j} x_{m}\right)\right]\right) \\
= & V\left(w, \alpha_{m, 0} \nabla g\left(x_{m}\right)+\sum_{j=1}^{\infty} \alpha_{m, j} \nabla g\left(T_{j}^{m} x_{m}\right)\right) \\
= & g(w)-\left\langle w, \alpha_{m, 0} \nabla g\left(x_{m}\right)+\sum_{j=1}^{\infty} \alpha_{m, j} \nabla g\left(T_{j} x_{m}\right)\right\rangle \\
& +g^{*}\left(\alpha_{m, 0} \nabla g\left(x_{m}\right)+\sum_{j=1}^{\infty} \alpha_{m, j} \nabla g\left(T_{j} x_{m}\right)\right) \\
\leq & \alpha_{m, 0} g(w)+\sum_{j=1}^{\infty} \alpha_{m, j} g(w)+\alpha_{m, 0} g^{*}\left(\nabla g\left(x_{m}\right)\right)+\sum_{j=1}^{\infty} \alpha_{m, j} g^{*}\left(\nabla g\left(T_{j} x_{m}\right)\right) \\
= & \alpha_{m, 0} V\left(w, \nabla g\left(x_{m}\right)\right)+\sum_{j=1}^{\infty} \alpha_{m, j} V\left(w, \nabla g\left(T_{j} x_{m}\right)\right) \\
= & \alpha_{m, 0} D_{g}\left(w, x_{m}\right)+\sum_{j=1}^{\infty} \alpha_{m, j} D_{g}\left(w, T_{j} x_{m}\right) \\
\leq & \alpha_{m, 0} D_{g}\left(w, x_{m}\right)+\sum_{j=1}^{\infty} \alpha_{m, j} D_{g}\left(w, x_{m}\right) \\
= & D_{g}\left(w, x_{m}\right) .
\end{aligned}
$$

This implies that

$$
\begin{aligned}
D_{g}\left(w, y_{m}\right) & =D_{g}\left(w, \nabla g^{*}\left[\beta_{m} \nabla g\left(x_{m}\right)+\left(1-\beta_{m}\right) \nabla g\left(z_{m}\right)\right]\right) \\
& =V\left(w, \beta_{m} \nabla g\left(x_{m}\right)+\left(1-\beta_{m}\right) \nabla g\left(z_{m}\right)\right) \\
& \leq \beta_{m} V\left(w, \nabla g\left(x_{m}\right)\right)+\left(1-\beta_{m}\right) V\left(w, \nabla\left(z_{m}\right)\right) \\
& =\beta_{m} D_{g}\left(w, x_{m}\right)+\left(1-\beta_{m}\right) D_{g}\left(w, z_{m}\right) \\
& \leq \beta_{m} D_{g}\left(w, x_{m}\right)+\left(1-\beta_{m}\right) D_{g}\left(w, x_{m}\right) \\
& =D_{g}\left(w, x_{m}\right) .
\end{aligned}
$$

This proves that $w \in C_{m+1}$. Thus, we have $F \subset C_{n}$ for all $n \in \mathbb{N} \cup\{0\}$. 
Step 3. We prove that $\left\{x_{n}\right\}_{n \in \mathbb{N}},\left\{y_{n}\right\}_{n \in \mathbb{N}},\left\{z_{n}\right\}_{n \in \mathbb{N}}$ and $\left\{T_{j} x_{n}: j, n \in \mathbb{N} \cup\{0\}\right\}$ are bounded sequences in $C$.

In view of (1.9), we conclude that

$$
\begin{aligned}
D_{g}\left(x_{n}, x\right) & =D_{g}\left(\operatorname{proj}_{C_{n}}^{g} x, x\right) \leq D_{g}(w, x)-D_{g}\left(w, x_{n}\right) \\
& \leq D_{g}(w, x), \quad \forall w \in F \subset C_{n}, n \in \mathbb{N} \cup\{0\} .
\end{aligned}
$$

This implies that the sequence $\left\{D\left(x_{n}, x\right)\right\}_{n \in \mathbb{N}}$ is bounded and hence there exists $M>0$ such that

$$
D_{g}\left(x_{n}, x\right) \leq M, \quad \forall n \in \mathbb{N} .
$$

In view of Lemma 2.1(3), we conclude that the sequence $\left\{x_{n}\right\}_{n \in \mathbb{N}}$ is bounded. Since $\left\{T_{j}\right\}_{j \in \mathbb{N}}$ is an infinite family of Bregman weak relatively nonexpansive mappings from $C$ into itself, we have for any $q \in F$ that

$$
D_{g}\left(q, T_{j} x_{n}\right) \leq D_{g}\left(q, x_{n}\right), \quad \forall j, n \in \mathbb{N} .
$$

This, together with Definition 2.1 and the boundedness of $\left\{x_{n}\right\}_{n \in \mathbb{N}}$, implies that the sequence $\left\{T_{j} x_{n}: j, n \in \mathbb{N} \cup\{0\}\right\}$ is bounded.

Step 4. We show that $x_{n} \rightarrow u$ for some $u \in F$, where $u=\operatorname{proj}_{F}^{g} x$.

By Step 3, we have that $\left\{x_{n}\right\}_{n \in \mathbb{N}}$ is bounded. By the construction of $C_{n}$, we conclude that $C_{m} \subset C_{n}$ and $x_{m}=\operatorname{proj}_{C_{m}}^{g} x \in C_{m} \subset C_{n}$ for any positive integer $m \geq n$. This, together with (1.9), implies that

$$
\begin{aligned}
D_{g}\left(x_{m}, x_{n}\right) & =D_{g}\left(x_{m}, \operatorname{proj}_{C_{n}}^{g} x\right) \leq D_{g}\left(x_{m}, x\right)-D_{g}\left(\operatorname{proj}_{C_{n}}^{g} x, x\right) \\
& =D_{g}\left(x_{m}, x\right)-D_{g}\left(x_{n}, x\right) .
\end{aligned}
$$

In view of (1.9), we conclude that

$$
\begin{aligned}
D_{g}\left(x_{n}, x\right) & =D_{g}\left(\operatorname{proj}_{C_{n}}^{g} x, x\right) \leq D_{g}(w, x)-D_{g}\left(w, x_{n}\right) \\
& \leq D_{g}(w, x), \quad \forall w \in F \subset C_{n}, n \in \mathbb{N} \cup\{0\} .
\end{aligned}
$$

It follows from (3.4) that the sequence $\left\{D_{g}\left(x_{n}, x\right)\right\}_{n \in \mathbb{N}}$ is bounded and hence there exists $M>0$ such that

$$
D_{g}\left(x_{n}, x\right) \leq M, \quad \forall n \in \mathbb{N} .
$$

In view of (3.3), we conclude that

$$
D_{g}\left(x_{n}, x\right) \leq D_{g}\left(x_{n}, x\right)+D_{g}\left(x_{m}, x_{n}\right) \leq D_{g}\left(x_{m}, x\right), \quad \forall m \geq n .
$$

This proves that $\left\{D_{g}\left(x_{n}, x\right)\right\}_{n \in \mathbb{N}}$ is an increasing sequence in $\mathbb{R}$ and hence by (3.5) the limit $\lim _{n \rightarrow \infty} D_{g}\left(x_{n}, x\right)$ exists. Letting $m, n \rightarrow \infty$ in (3.3), we deduce that $D_{g}\left(x_{m}, x_{n}\right) \rightarrow 0$. In view of Lemma 2.4, we get that $\left\|x_{m}-x_{n}\right\| \rightarrow 0$ as $m, n \rightarrow \infty$. This means that $\left\{x_{n}\right\}_{n \in \mathbb{N}}$ is a Cauchy 
sequence. Since $E$ is a Banach space and $C$ is closed and convex, we conclude that there exists $u \in C$ such that

$$
\lim _{n \rightarrow \infty}\left\|x_{n}-u\right\|=0
$$

Now, we show that $u \in F$. In view of (3.3), we obtain

$$
\lim _{n \rightarrow \infty} D_{g}\left(x_{n+1}, x_{n}\right)=0
$$

Since $x_{n+1} \in C_{n+1}$, we conclude that

$$
D_{g}\left(x_{n+1}, y_{n}\right) \leq D_{g}\left(x_{n+1}, x_{n}\right)
$$

This, together with (3.7), implies that

$$
\lim _{n \rightarrow \infty} D_{g}\left(x_{n+1}, y_{n}\right)=0
$$

Employing Lemma 2.4 and (3.7)-(3.8), we deduce that

$$
\lim _{n \rightarrow \infty}\left\|x_{n+1}-x_{n}\right\|=0 \text { and } \quad \lim _{n \rightarrow \infty}\left\|x_{n+1}-y_{n}\right\|=0 \text {. }
$$

In view of (3.6), we get

$$
\lim _{n \rightarrow \infty}\left\|y_{n}-u\right\|=0
$$

From (3.6) and (3.9), it follows that

$$
\lim _{n \rightarrow \infty}\left\|x_{n}-y_{n}\right\|=0 \text {. }
$$

Since $\nabla g$ is uniformly norm-to-norm continuous on any bounded subset of $E$, we obtain

$$
\lim _{n \rightarrow \infty}\left\|\nabla g\left(x_{n}\right)-\nabla g\left(y_{n}\right)\right\|=0 \text {. }
$$

In view of (3.1), we have

$$
\nabla g\left(y_{n}\right)-\nabla g\left(x_{n}\right)=\left(1-\beta_{n}\right)\left(\nabla g\left(z_{n}\right)-\nabla g\left(x_{n}\right)\right) \text {. }
$$

It follows from (3.10)-(3.11) that

$$
\lim _{n \rightarrow \infty}\left\|\nabla g\left(z_{n}\right)-\nabla g\left(x_{n}\right)\right\|=0 \text {. }
$$

Since $\nabla g$ is uniformly norm-to-norm continuous on any bounded subset of $E$, we obtain

$$
\lim _{n \rightarrow \infty}\left\|z_{n}-x_{n}\right\|=0 .
$$

Applying Lemma 2.4, we derive that

$$
\lim _{n \rightarrow \infty} D_{g}\left(z_{n}, x_{n}\right)=0 \text {. }
$$


It follows from the three point identity (see (2.2)) that

$$
\begin{aligned}
\left|D_{g}\left(w, x_{n}\right)-D_{g}\left(w, z_{n}\right)\right|= & \mid D_{g}\left(w, z_{n}\right)+D_{g}\left(z_{n}, x_{n}\right) \\
& +\left\langle w-z_{n}, \nabla g\left(z_{n}\right)-\nabla g\left(x_{n}\right)\left|-D_{g}\left(w, z_{n}\right)\right|\right. \\
= & \left|D_{g}\left(z_{n}, x_{n}\right)-\left\langle w-z_{n}, \nabla g\left(z_{n}\right)-\nabla g\left(x_{n}\right)\right\rangle\right| \\
\leq & D_{g}\left(z_{n}, x_{n}\right)+\left\|w-z_{n}\right\|\left\|\nabla g\left(z_{n}\right)-\nabla g\left(x_{n}\right)\right\| \\
\rightarrow & 0
\end{aligned}
$$

as $n \rightarrow \infty$.

The function $g$ is bounded on bounded subsets of $E$ and thus $\nabla g$ is also bounded on bounded subsets of $E^{*}$ (see, for example, [23, Proposition 1.1.11] for more details). This implies that the sequences $\left\{\nabla g\left(x_{n}\right)\right\}_{n \in \mathbb{N}},\left\{\nabla g\left(y_{n}\right)\right\}_{n \in \mathbb{N}},\left\{\nabla g\left(z_{n}\right)\right\}_{n \in \mathbb{N}}$ and $\left\{\nabla g\left(T_{j}^{n} x_{n}\right): n, j \in\right.$ $\mathbb{N} \cup\{0\}\}$ are bounded in $E^{*}$.

In view of Theorem 2.2(3), we know that $\operatorname{dom} g^{*}=E^{*}$ and $g^{*}$ is strongly coercive and uniformly convex on bounded subsets. Let $s=\sup \left\{\left\|\nabla g\left(T_{j}^{n} x_{n}\right)\right\|: j \in \mathbb{N} \cup\{0\}, n \in \mathbb{N} \cup\{0\}\right\}$ and $\rho_{s}^{*}: E^{*} \rightarrow \mathbb{R}$ be the gauge of uniform convexity of the conjugate function $g^{*}$. Now, we fix $i \in \mathbb{N}$ satisfying condition (2). We prove that for any $w \in F$ and $j \in \mathbb{N} \cup\{0\}$

$$
D_{g}\left(w, z_{n}\right) \leq D_{g}\left(w, x_{n}\right)-\alpha_{n, i} \alpha_{n, j} \rho_{s}^{*}\left(\left\|\nabla g\left(T_{i} x_{n}\right)-\nabla g\left(T_{j} x_{n}\right)\right\|\right) .
$$

Let us show (3.14). For any given $w \in F(T)$ and $j \in \mathbb{N}$, in view of the definition of the Bregman distance (see (1.7)), (1.6), Lemmas 2.3 and 2.5, we obtain

$$
\begin{aligned}
D_{g}\left(w, z_{n}\right)= & D_{g}\left(w, \nabla g^{*}\left[\alpha_{n, 0} \nabla g\left(x_{n}\right)+\sum_{j=1}^{\infty} \alpha_{n, j} \nabla g\left(T_{j} x_{n}\right)\right]\right) \\
= & V\left(w, \alpha_{n, 0} \nabla g\left(x_{n}\right)+\sum_{j=1}^{\infty} \alpha_{n, j} \nabla g\left(T_{j} x_{n}\right)\right) \\
= & g(w)-\left\langle w, \alpha_{n, 0} \nabla g\left(x_{n}\right)+\sum_{j=1}^{\infty} \alpha_{n, j} \nabla g\left(T_{j} x_{n}\right)\right\rangle \\
& +g^{*}\left(\alpha_{n, 0} \nabla g\left(x_{n}\right)+\sum_{j=1}^{\infty} \alpha_{n, j} \nabla g\left(T_{j} x_{n}\right)\right) \\
\leq & \alpha_{n, 0} g(w)+\sum_{j=1}^{\infty} \alpha_{n, j} g(w) \\
& -\alpha_{n, 0}\left\langle w, \nabla g\left(x_{n}\right)\right\rangle-\sum_{j=1}^{\infty} \alpha_{n, j}\left\langle w, \nabla g\left(T_{j} x_{n}\right)\right\rangle \\
& +\alpha_{n, 0} g^{*}\left(\nabla g\left(x_{n}\right)\right)+\sum_{j=1}^{\infty} \alpha_{n, j} g^{*}\left(\nabla g\left(T_{j} x_{n}\right)\right) \\
& -\alpha_{n, i} \alpha_{n, j} \rho_{s}^{*}\left(\left\|\nabla g\left(T_{i} x_{n}\right)-\nabla g\left(T_{j} x_{n}\right)\right\|\right) \\
= & \alpha_{n, 0} V\left(w, \nabla g\left(x_{n}\right)\right)+\sum_{j=1}^{\infty} \alpha_{n, j} V\left(w, \nabla g\left(T_{j} x_{n}\right)\right)
\end{aligned}
$$




$$
\begin{aligned}
& -\alpha_{n, i} \alpha_{n, j} \rho_{s}^{*}\left(\left\|\nabla g\left(T_{i} x_{n}\right)-\nabla g\left(T_{j} x_{n}\right)\right\|\right) \\
= & \alpha_{n, 0} D_{g}\left(w, x_{n}\right)+\sum_{j=1}^{\infty} \alpha_{n, j} D_{g}\left(w, T_{j} x_{n}\right)-\alpha_{n, i} \alpha_{n, j} \rho_{s}^{*}\left(\left\|\nabla g\left(T_{i} x_{n}\right)-\nabla g\left(T_{j} x_{n}\right)\right\|\right) \\
\leq & \alpha_{n, 0} D_{g}\left(w, x_{n}\right)+\sum_{j=1}^{\infty} \alpha_{n, j} D_{g}\left(w, x_{n}\right)-\alpha_{n, i} \alpha_{n, j} \rho_{s}^{*}\left(\left\|\nabla g\left(T_{i} x_{n}\right)-\nabla g\left(T_{j} x_{n}\right)\right\|\right) \\
= & D_{g}\left(w, x_{n}\right)-\alpha_{n, i} \alpha_{n, j} \rho_{s}^{*}\left(\left\|\nabla g\left(T_{i} x_{n}\right)-\nabla g\left(T_{j} x_{n}\right)\right\|\right) .
\end{aligned}
$$

In view of (3.13), we obtain

$$
D_{g}\left(w, x_{n}\right)-D_{g}\left(w, z_{n}\right) \rightarrow 0 \quad \text { as } n \rightarrow \infty
$$

In view of (3.14) and (3.15), we conclude that

$$
\alpha_{n, i} \alpha_{n, j} \rho_{s}^{*}\left(\left\|\nabla g\left(T_{i} x_{n}\right)-\nabla g\left(T_{j} x_{n}\right)\right\|\right) \leq D_{g}\left(w, x_{n}\right)-D_{g}\left(w, z_{n}\right) \rightarrow 0
$$

as $n \rightarrow \infty$. From the assumption $\liminf _{n \rightarrow \infty} \alpha_{n, i} \alpha_{n, j}>0, \forall j \in \mathbb{N} \cup\{0\}$, we have

$$
\lim _{n \rightarrow \infty} \rho_{s}^{*}\left(\left\|\nabla g\left(T_{i} x_{n}\right)-\nabla g\left(T_{j} x_{n}\right)\right\|\right)=0, \quad \forall j \in \mathbb{N} \cup\{0\}
$$

Therefore, from the property of $\rho_{s}^{*}$, we deduce that

$$
\lim _{n \rightarrow \infty}\left\|\nabla g\left(T_{i} x_{n}\right)-\nabla g\left(T_{j} x_{n}\right)\right\|=0, \quad \forall j \in \mathbb{N} \cup\{0\}
$$

Since $\nabla g^{*}$ is uniformly norm-to-norm continuous on bounded subsets of $E^{*}$, we arrive at

$$
\lim _{n \rightarrow \infty}\left\|T_{i} x_{n}-T_{j} x_{n}\right\|=0, \quad \forall j \in \mathbb{N} \cup\{0\}
$$

In particular, for $j=0$, we have

$$
\lim _{n \rightarrow \infty}\left\|T_{i} x_{n}-x_{n}\right\|=0
$$

This, together with (3.16), implies that

$$
\lim _{n \rightarrow \infty}\left\|T_{j} x_{n}-x_{n}\right\|=0, \quad \forall j \in \mathbb{N} \cup\{0\}
$$

Since $\left\{T_{j}\right\}_{j \in \mathbb{N}}$ is an infinite family of Bregman weak relatively nonexpansive mappings, from (3.6) and (3.17), we conclude that $T_{j} u=u, \forall j \in \mathbb{N} \cup\{0\}$. Thus, we have $u \in F$.

Finally, we show that $u=\operatorname{proj}_{F}^{g} x$. From $x_{n}=\operatorname{proj}_{C_{n}}^{g} x$, we conclude that

$$
\left\langle z-x_{n}, \nabla g\left(x_{n}\right)-\nabla g(x)\right\rangle \geq 0, \quad \forall z \in C_{n} .
$$

Since $F \subset C_{n}$ for each $n \in \mathbb{N}$, we obtain

$$
\left\langle z-x_{n}, \nabla g\left(x_{n}\right)-\nabla g(x)\right\rangle \geq 0, \quad \forall z \in F
$$


Letting $n \rightarrow \infty$ in (3.18), we deduce that

$$
\langle z-u, \nabla g(u)-\nabla g(x)| \geq 0, \quad \forall z \in F .
$$

In view of (1.8), we have $u=\operatorname{proj}_{F}^{g} x$, which completes the proof.

Remark 3.1 Theorem 3.1 improves Theorem 1.2 in the following aspects.

(1) For the structure of Banach spaces, we extend the duality mapping to a more general case, that is, a convex, continuous and strongly coercive Bregman function which is bounded on bounded subsets and uniformly convex and uniformly smooth on bounded subsets.

(2) For the mappings, we extend the mapping from a relatively nonexpansive mapping to a countable family of Bregman weak relatively nonexpansive mappings. We remove the assumption $\hat{F}(T)=F(T)$ on the mapping $T$ and extend the result to a countable family of Bregman weak relatively nonexpansive mappings, where $\hat{F}(T)$ is the set of asymptotic fixed points of the mapping $T$.

(3) For the algorithm, we remove the set $W_{n}$ in Theorem 1.2.

Lemma 3.2 Let $E$ be a reflexive Banach space and let $g: E \rightarrow \mathbb{R}$ be a strongly coercive Bregman function which is bounded on bounded subsets and uniformly convex and uniformly smooth on bounded subsets of $E$. Let $A$ be a maximal monotone operator from $E$ to $E^{*}$ such that $A^{-1}(0) \neq \varnothing$. Let $r>0$ and $\operatorname{Res}_{r A}^{g}=(\nabla g+r A)^{-1} \nabla g$ be the $g$-resolvent of $A$. Then $\operatorname{Res}_{r A}^{g}$ is a Bregman weak relatively nonexpansive mapping.

Proof Let $\left\{z_{n}\right\}_{n \in \mathbb{N}} \subset E$ be a sequence such that $z_{n} \rightarrow z$ and $\lim _{n \rightarrow \infty}\left\|z_{n}-\operatorname{Res}_{r A}^{g} z_{n}\right\|=0$. Since $\nabla g$ is uniformly norm-to-norm continuous on bounded subsets of $E$, we obtain

$$
\frac{1}{r}\left(\nabla g\left(z_{n}\right)-\nabla g\left(\operatorname{Res}_{r A}^{g} z_{n}\right)\right) \rightarrow 0
$$

It follows from

$$
\frac{1}{r}\left(\nabla g\left(z_{n}\right)-\nabla g\left(\operatorname{Res}_{r A}^{g} z_{n}\right)\right) \in A \operatorname{Res}_{r A}^{g} z_{n}
$$

and the monotonicity of $A$ that

$$
\left\langle w-\operatorname{Res}_{r A}^{g} z_{n}, y-\frac{1}{r}\left(\nabla g\left(z_{n}\right)-\nabla g\left(\operatorname{Res}_{r A}^{g} z_{n}\right)\right)\right\rangle \geq 0
$$

for all $w \in \operatorname{dom} A$ and $y \in A w$. Letting $n \rightarrow \infty$ in the above inequality, we have $\langle w-z, y\rangle \geq 0$ for all $w \in \operatorname{dom} A$ and $y \in A w$. Therefore, from the maximality of $A$, we conclude that $z \in A^{-1}(0)=F\left(\operatorname{Res}_{r A}^{g}\right)$, that is, $z=\operatorname{Res}_{r A}^{g} z$. Hence $\operatorname{Res}_{r A}^{g}$ is Bregman weak relatively nonexpansive, which completes the proof.

As an application of our main result, we include a concrete example in support of Theorem 3.1. Using Theorem 3.1, we obtain the following strong convergence theorem for maximal monotone operators. 
Theorem 3.2 Let $E$ be a reflexive Banach space and let $g: E \rightarrow \mathbb{R}$ be a strongly coercive Bregman function which is bounded on bounded subsets and uniformly convex and uniformly smooth on bounded subsets of $E$. Let $A$ be a maximal monotone operator from $E$ to $E^{*}$ such that $A^{-1}(0) \neq \varnothing$. Let $r_{n}>0$ such that $\liminf _{n \rightarrow \infty} r_{n}>0$ and $\operatorname{Res}_{r_{n} A}^{g}=\left(\nabla g+r_{n} A\right)^{-1} \nabla g$ be the g-resolvent of $A$. Let $\left\{x_{n}\right\}_{n \in \mathbb{N}}$ be a sequence generated by

$$
\left\{\begin{array}{l}
x_{0}=x \in C \quad \text { chosen arbitrarily, } \\
C_{0}=C, \\
z_{n}=\nabla g^{*}\left[\alpha_{n, 0} \nabla g\left(x_{n}\right)+\sum_{j=1}^{\infty} \alpha_{n, j} \nabla g\left(\operatorname{Res}_{r_{j} A}^{g} x_{n}\right)\right], \\
y_{n}=\nabla g^{*}\left[\beta_{n} \nabla g\left(x_{n}\right)+\left(1-\beta_{n}\right) \nabla g\left(z_{n}\right)\right], \\
C_{n+1}=\left\{z \in C_{n}: D_{g}\left(z, y_{n}\right) \leq D_{g}\left(z, x_{n}\right)\right\}, \\
x_{n+1}=\operatorname{proj}_{C_{n+1}}^{g} x \quad \text { and } \quad n \in \mathbb{N} \cup\{0\},
\end{array}\right.
$$

where $\nabla g$ is the right-hand derivative of $g$. Let $\left\{\alpha_{n, j}: j, n \in \mathbb{N} \cup\{0\}\right\}$ and $\left\{\beta_{n}\right\}_{n \in \mathbb{N} \cup\{0\}}$ be sequences in $[0,1)$ satisfying the following control conditions:

(1) $\sum_{j=0}^{\infty} \alpha_{n, j}=1, \forall n \in \mathbb{N} \cup\{0\}$;

(2) There exists $i \in \mathbb{N}$ such that $\liminf _{n \rightarrow \infty} \alpha_{n, i} \alpha_{n, j}>0, \forall j \in \mathbb{N} \cup\{0\}$;

(3) $0 \leq \beta_{n}<1$ for all $n \in \mathbb{N} \cup\{0\}$ and $\liminf _{n \rightarrow \infty} \beta_{n}<1$.

Then the sequence $\left\{x_{n}\right\}_{n \in \mathbb{N}}$ defined in (3.19) converges strongly to proj $_{A^{-1}(0)}^{g} x$ as $n \rightarrow \infty$.

Proof Letting $T_{j}=\operatorname{Res}_{r_{j} A}^{g}, \forall j \in \mathbb{N} \cup\{0\}$, in Theorem 3.1, from (3.1) we obtain (3.19). We need only to show that $T_{j}$ satisfies all the conditions in Theorem 3.1 for all $j \in \mathbb{N} \cup\{0\}$. In view of Lemma 3.2, we conclude that $T_{j}$ is a Bregman relatively nonexpansive mapping for each $j \in \mathbb{N} \cup\{0\}$. Thus, we obtain

$$
D_{g}\left(p, \operatorname{Res}_{r_{j} A}^{g} v\right) \leq D_{g}(p, v), \quad \forall v \in E, p \in F\left(\operatorname{Res}_{r_{j} A}^{g}\right), \forall j \in \mathbb{N} \cup\{0\}
$$

and

$$
\tilde{F}\left(\operatorname{Res}_{r_{j} A}^{g}\right)=F\left(\operatorname{Res}_{r_{j} A}^{g}\right)=A^{-1}(0), \quad \forall j \in \mathbb{N} \cup\{0\},
$$

where $\tilde{F}\left(\operatorname{Res}_{r_{j} A}^{g}\right)$ is the set of all strong asymptotic fixed points of $\operatorname{Res}_{r_{j} A}^{g}$. Therefore, in view of Theorem 3.1, we have the conclusions of Theorem 3.2. This completes the proof.

\section{Strong convergence theorems with computational errors}

In this section, we study strong convergence of iterative algorithms to find common fixed points of finitely many Bregman weak relatively nonexpansive mappings in a reflexive Banach space. Our algorithms take into account possible computational errors. We prove the following strong convergence theorem concerning Bregman weak relatively nonexpansive mappings.

Theorem 4.1 Let $E$ be a reflexive Banach space and let $g: E \rightarrow \mathbb{R}$ be a strongly coercive Bregman function which is bounded on bounded subsets and uniformly convex and uniformly smooth on bounded subsets of $E$. Let $N \in \mathbb{N}$ and $\left\{T_{j}\right\}_{j=1}^{N}$ be a finite family of Bregman weak relatively nonexpansive mappings from $E$ into int $\operatorname{dom} g$ such that $F:=\bigcap_{j=1}^{N} F\left(T_{j}\right)$ is 
a nonempty subset of $E$. Suppose in addition that $T_{0}=I$, where $I$ is the identity mapping on E. Let $\left\{x_{n}\right\}_{n \in \mathbb{N}}$ be a sequence generated by

$$
\left\{\begin{aligned}
& x_{0}=x \in E \quad \text { chosen arbitrarily, } \\
& C_{0}=E, \\
& y_{n}=\nabla g^{*}\left[\alpha_{n, 0} \nabla g\left(x_{n}\right)+\sum_{j=1}^{N} \alpha_{n, j} \nabla g\left(T_{j}\left(x_{n}+e_{n}^{j}\right)\right)\right], \\
& C_{n+1}=\left\{z \in C_{n}: D_{g}\left(z, y_{n}\right) \leq D_{g}\left(z, x_{n}\right)+\sum_{j=1}^{N} \alpha_{n, j} D_{g}\left(x_{n}, x_{n}+e_{n}^{j}\right)\right. \\
&\left.+\sum_{j=1}^{N} \alpha_{n, j}\left\langle z-x_{n}, \nabla g\left(x_{n}\right)-\nabla g\left(x_{n}+e_{n}^{j}\right)\right\rangle\right\} \\
& x_{n+1}= \operatorname{proj}_{C_{n+1}}^{g} x \text { and } n \in \mathbb{N} \cup\{0\},
\end{aligned}\right.
$$

where $\nabla g$ is the right-hand derivative of $g$. Let $\left\{\alpha_{n, j}: n \in \mathbb{N} \cup\{0\}, j \in\{0,1,2, \ldots, N\}\right\}$ be a sequence in $(0,1)$ satisfying the following control conditions:

(1) $\sum_{j=0}^{N} \alpha_{n, j}=1, \forall n \in \mathbb{N} \cup\{0\}$;

(2) There exists $i \in\{1,2, \ldots, N\}$ such that $\liminf _{n \rightarrow \infty} \alpha_{n, i} \alpha_{n, j}>0, \forall j \in\{0,1,2, \ldots, N\}$.

If, for each $j=0,1,2, \ldots, N$, the sequences of errors $\left\{e_{n}^{j}\right\}_{n \in \mathbb{N}} \subset E$ satisfy $\liminf _{n \rightarrow \infty} e_{n}^{j}=0$, then the sequence $\left\{x_{n}\right\}_{n \in \mathbb{N}}$ defined in (4.1) converges strongly to $\operatorname{proj}_{F}^{g} x$ as $n \rightarrow \infty$.

Proof We divide the proof into several steps.

Step 1 . We show that $C_{n}$ is closed and convex for each $n \in \mathbb{N} \cup\{0\}$.

It is clear that $C_{0}=E$ is closed and convex. Let $C_{m}$ be closed and convex for some $m \in \mathbb{N}$. For $z \in C_{m}$, we see that

$$
\begin{aligned}
D_{g}\left(z, y_{m}\right) \leq & D_{g}\left(z, x_{m}\right)+\sum_{j=1}^{N} \alpha_{m, j} D_{g}\left(x_{m}, x_{m}+e_{m}^{j}\right) \\
& \left.+\sum_{j=1}^{N} \alpha_{m, j} \mid z-x_{m}, \nabla g\left(x_{m}\right)-\nabla g\left(x_{m}+e_{m}^{j}\right)\right)
\end{aligned}
$$

is equivalent to

$$
\begin{aligned}
& \left\langle z, \nabla g\left(x_{m}\right)-\nabla g\left(y_{m}\right)\right\rangle+\sum_{j=1}^{N} \alpha_{m, j}\left\langle x_{m}-z, \nabla g\left(x_{m}\right)-\nabla g\left(x_{m}+e_{m}^{j}\right)\right\rangle \\
& \quad \leq g\left(y_{m}\right)-g\left(x_{m}\right)+\left\langle x_{m}, \nabla g\left(x_{m}\right)\right\rangle-\left\langle y_{m}, \nabla g\left(y_{m}\right)\right\rangle+\sum_{j=1}^{N} \alpha_{m, j} D_{g}\left(x_{m}, x_{m}+e_{m}^{j}\right) .
\end{aligned}
$$

An easy argument shows that $C_{m+1}$ is closed and convex. Hence $C_{n}$ is closed and convex for all $n \in \mathbb{N} \cup\{0\}$.

Step 2. We claim that $F \subset C_{n}$ for all $n \in \mathbb{N} \cup\{0\}$.

It is obvious that $F \subset C_{0}=E$. Assume now that $F \subset C_{m}$ for some $m \in \mathbb{N}$. Employing Lemma 2.5, for any $w \in F \subset C_{m}$, we obtain

$$
\begin{aligned}
D_{g}\left(w, y_{m}\right) & =D_{g}\left(w, \nabla g^{*}\left[\alpha_{m, 0} \nabla g\left(x_{m}\right)+\sum_{j=1}^{N} \alpha_{m, j} \nabla g\left(T_{j}\left(x_{m}+e_{m}^{j}\right)\right)\right]\right) \\
& =V\left(w, \alpha_{m, 0} \nabla g\left(x_{m}\right)+\sum_{j=1}^{N} \alpha_{m, j} \nabla g\left(T_{j}\left(x_{m}+e_{m}^{j}\right)\right)\right)
\end{aligned}
$$




$$
\begin{aligned}
= & g(w)-\left\langle w, \alpha_{m, 0} \nabla g\left(x_{m}\right)+\sum_{j=1}^{N} \alpha_{m, j} \nabla g\left(T_{j}\left(x_{m}+e_{m}^{j}\right)\right)\right\rangle \\
& +g^{*}\left(\alpha_{m, 0} \nabla g\left(x_{m}\right)\right)+\sum_{j=1}^{N} \alpha_{m, j} \nabla g\left(T_{j}\left(x_{m}+e_{m}^{j}\right)\right) \\
\leq & \alpha_{m, 0} g(w)+\sum_{j=1}^{N} \alpha_{m, j} g(w) \\
& +\alpha_{m, 0} g^{*}\left(\nabla g\left(x_{m}\right)\right)+\sum_{j=1}^{N} \alpha_{m, j} g^{*}\left(\nabla g\left(T_{j}\left(x_{m}+e_{m}^{j}\right)\right)\right) \\
& +\alpha_{m, 0} V\left(w, \nabla g\left(x_{m}\right)\right)+\sum_{j=1}^{N} \alpha_{m, j} V\left(w, \nabla g\left(T_{j}\left(x_{m}+e_{m}^{j}\right)\right)\right) \\
& +\sum_{j=1}^{N} \alpha_{m, j}\left\langle w-x_{m}, \nabla g\left(x_{m}\right)-\nabla g\left(x_{m}+e_{m}^{j}\right)\right\rangle \\
= & \alpha_{m, 0} D_{g}\left(w, x_{m}\right)+\sum_{j=1}^{N} \alpha_{m, j} D_{g}\left(w, T_{j}\left(x_{m}+e_{m}^{j}\right)\right) \\
\leq & \alpha_{m, 0} D_{g}\left(w, x_{m}\right)+\sum_{j=1}^{N} \alpha_{m, j} D_{g}\left(w, x_{m}\right)+\sum_{j=1}^{N} \alpha_{m, j} D_{g}\left(x_{m}, x_{m}+e_{m}^{j}\right) \\
\leq & \alpha_{m, 0} D_{g}\left(w, x_{m}\right)+\sum_{j=1}^{N} \alpha_{m, j} D_{g}\left(w, x_{m}+e_{m}^{j}\right) \\
& \\
& \\
& \\
&
\end{aligned}
$$

This proves that $w \in C_{m+1}$. Consequently, we see that $F \subset C_{n}$ for any $n \in \mathbb{N} \cup\{0\}$.

Step 3. We prove that $\left\{x_{n}\right\}_{n \in \mathbb{N}},\left\{y_{n}\right\}_{n \in \mathbb{N}}$ and $\left\{T_{j}\left(x_{n}+e_{n}^{j}\right): n \in \mathbb{N}, j \in\{0,1,2, \ldots, N\}\right\}$ are bounded sequences in $E$.

In view of (1.9), we conclude that

$$
\begin{aligned}
D_{g}\left(x_{n}, x\right) & =D_{g}\left(\operatorname{proj}_{C_{n}}^{g} x, x\right) \leq D_{g}(w, x)-D_{g}\left(w, x_{n}\right) \\
& \leq D_{g}(w, x), \quad \forall w \in F \subset C_{n}, n \in \mathbb{N} \cup\{0\}
\end{aligned}
$$

It follows from (4.3) that the sequence $\left\{D_{g}\left(x_{n}, x\right)\right\}_{n \in \mathbb{N}}$ is bounded and hence there exists $M_{0}>0$ such that

$$
D_{g}\left(x_{n}, x\right) \leq M_{0}, \quad \forall n \in \mathbb{N} \cup\{0\}
$$

In view of Lemma 2.1(3), we conclude that the sequence $\left\{x_{n}\right\}_{n \in \mathbb{N}}$ and hence $\left\{x_{n}+e_{n}^{j}: n \in\right.$ $\mathbb{N} \cup\{0\}, j \in\{0,1,2, \ldots, N\}\}$ is bounded. Since $\left\{T_{j}\right\}_{j=1}^{N}$ is a finite family of Bregman weak 
relatively nonexpansive mappings from $E$ into int $\operatorname{dom} g$, for any $q \in F$, we have

$$
D_{g}\left(q, T_{j}\left(x_{n}+e_{n}^{j}\right)\right) \leq D_{g}\left(q, x_{n}+e_{n}^{j}\right), \quad \forall n \in \mathbb{N} \text { and } j \in\{0,1,2, \ldots, N\} .
$$

This, together with Definition 2.1 and the boundedness of $\left\{x_{n}\right\}_{n \in \mathbb{N}}$, implies that $\left\{T_{j}\left(x_{n}+e_{n}^{j}\right)\right.$ : $n \in \mathbb{N} \cup\{0\}, j \in\{0,1,2, \ldots, N\}\}$ is bounded.

Step 4. We show that $x_{n} \rightarrow u$ for some $u \in F$, where $u=\operatorname{proj}_{F}^{g} x$.

By Step 3, we deduce that $\left\{x_{n}\right\}_{n \in \mathbb{N}}$ is bounded. By the construction of $C_{n}$, we conclude that $C_{m} \subset C_{n}$ and $x_{m}=\operatorname{proj}_{C_{m}}^{g} x \in C_{m} \subset C_{n}$ for any positive integer $m \geq n$. This, together with (1.9), implies that

$$
\begin{aligned}
D_{g}\left(x_{m}, x_{n}\right) & =D_{g}\left(x_{m}, \operatorname{proj}_{C_{n}}^{g} x\right) \leq D_{g}\left(x_{m}, x\right)-D_{g}\left(\operatorname{proj}_{C_{n}}^{g} x, x\right) \\
& =D_{g}\left(x_{m}, x\right)-D_{g}\left(x_{n}, x\right) .
\end{aligned}
$$

In view of (4.6), we have

$$
D_{g}\left(x_{n}, x\right) \leq D_{g}\left(x_{n}, x\right)+D_{g}\left(x_{m}, x_{n}\right) \leq D_{g}\left(x_{m}, x\right), \quad \forall m \geq n .
$$

This proves that $\left\{D_{g}\left(x_{n}, x\right)\right\}_{n \in \mathbb{N}}$ is an increasing sequence in $\mathbb{R}$ and hence by (4.4) the limit $\lim _{n \rightarrow \infty} D_{g}\left(x_{n}, x\right)$ exists. Letting $m, n \rightarrow \infty$ in (4.6), we deduce that $D_{g}\left(x_{m}, x_{n}\right) \rightarrow 0$. In view of Lemma 2.4, we obtain that $\left\|x_{m}-x_{n}\right\| \rightarrow 0$ as $m, n \rightarrow \infty$. Thus we have $\left\{x_{n}\right\}_{n \in \mathbb{N}}$ is a Cauchy sequence. Since $E$ is a Banach space, we conclude that there exists $u \in E$ such that

$$
\lim _{n \rightarrow \infty}\left\|x_{n}-u\right\|=0
$$

Now, we show that $u \in F$. In view of (4.6), we obtain

$$
\lim _{n \rightarrow \infty} D_{g}\left(x_{n+1}, x_{n}\right)=0
$$

Since $\lim _{n \rightarrow \infty} e_{n}^{j}=0$, for all $j \in\{0,1,2, \ldots, N\}$, in view of Lemma 2.4 and (4.8), we obtain that

$$
\lim _{n \rightarrow \infty}\left\|x_{n+1}-x_{n}\right\|=0 \quad \text { and } \quad \lim _{n \rightarrow \infty} D\left(x_{n}, x_{n}+e_{n}^{j}\right)=0, \quad j \in\{0,1,2, \ldots, N\} .
$$

The function $g$ is bounded on bounded subsets of $E$ and thus $\nabla g$ is also bounded on bounded subsets of $E^{*}$ (see, for example, [23, Proposition 1.1.11] for more details). Since $x_{n+1} \in C_{n+1}$, we get

$$
\begin{aligned}
D_{g}\left(x_{n+1}, y_{n}\right) \leq & D_{g}\left(x_{n+1}, x_{n}\right)+\sum_{j=1}^{N} \alpha_{n, j} D_{g}\left(x_{n}, x_{n}+e_{n}^{j}\right) \\
& +\sum_{j=1}^{N} \alpha_{n, j}\left(x_{n+1}-x_{n}, \nabla g\left(x_{n}\right)-\nabla g\left(x_{n}+e_{n}^{j}\right)\right\rangle .
\end{aligned}
$$

This, together with (4.9), implies that

$$
\lim _{n \rightarrow \infty} D_{g}\left(x_{n+1}, y_{n}\right)=0
$$


Employing Lemma 2.4 and (4.9)-(4.10), we deduce that

$$
\lim _{n \rightarrow \infty}\left\|x_{n+1}-y_{n}\right\|=0 .
$$

In view of (4.7) and (4.11), we get

$$
\lim _{n \rightarrow \infty}\left\|y_{n}-u\right\|=0
$$

Thus, $\left\{y_{n}\right\}_{n \in \mathbb{N}}$ is a bounded sequence.

From (4.11) and (4.12), it follows that

$$
\lim _{n \rightarrow \infty}\left\|x_{n}-y_{n}\right\|=0 .
$$

Since $\nabla g$ is uniformly norm-to-norm continuous on any bounded subset of $E$, we obtain

$$
\lim _{n \rightarrow \infty}\left\|\nabla g\left(x_{n}\right)-\nabla g\left(y_{n}\right)\right\|=0 .
$$

Applying Lemma 2.4, we deduce that

$$
\lim _{n \rightarrow \infty} D_{g}\left(y_{n}, x_{n}\right)=0 .
$$

It follows from the three point identity (see (2.2)) that

$$
\begin{aligned}
\left|D_{g}\left(w, x_{n}\right)-D_{g}\left(w, y_{n}\right)\right|= & \mid D_{g}\left(w, y_{n}\right)+D_{g}\left(y_{n}, x_{n}\right) \\
& +\left\langle w-y_{n}, \nabla g\left(y_{n}\right)-\nabla g\left(x_{n}\right)\right\rangle-D_{g}\left(w, y_{n}\right) \mid \\
= & \left|D_{g}\left(y_{n}, x_{n}\right)-\left\langle w-y_{n}, \nabla g\left(y_{n}\right)-\nabla g\left(x_{n}\right)\right\rangle\right| \\
\leq & D_{g}\left(y_{n}, x_{n}\right)+\left\|w-y_{n}\right\|\left\|\nabla g\left(y_{n}\right)-\nabla g\left(x_{n}\right)\right\| \\
\rightarrow & 0
\end{aligned}
$$

as $n \rightarrow \infty$.

The function $g$ is bounded on bounded subsets of $E$ and thus $\nabla g$ is also bounded on bounded subsets of $E^{*}$ (see, for example, [23, Proposition 1.1.11] for more details). This, together with Step 3, implies that the sequences $\left\{\nabla g\left(x_{n}\right)\right\}_{n \in \mathbb{N}},\left\{\nabla g\left(y_{n}\right)\right\}_{n \in \mathbb{N}}$ and $\left\{\nabla g\left(T_{j}\left(x_{n}+\right.\right.\right.$ $\left.\left.\left.e_{n}^{j}\right)\right): n \in \mathbb{N} \cup\{0\}, j \in\{0,1,2, \ldots, N\}\right\}$ are bounded in $E^{*}$.

In view of Theorem 2.2(3), we know that dom $g^{*}=E^{*}$ and $g^{*}$ is strongly coercive and uniformly convex on bounded subsets. Let $s=\sup \left\{\left\|\nabla g\left(T_{j}\left(x_{n}+e_{n}^{j}\right)\right)\right\|: j \in\{0,1,2, \ldots, N\}, n \in\right.$ $\mathbb{N} \cup\{0\}\}$ and let $\rho_{s}^{*}: E^{*} \rightarrow \mathbb{R}$ be the gauge of uniform convexity of the conjugate function $g^{*}$. Suppose that $i \in \mathbb{N}$ satisfies condition (2). We prove that for any $w \in F$ and $j \in\{0,1,2, \ldots, N\}$,

$$
\begin{aligned}
D_{g}\left(w, y_{n}\right) \leq & D_{g}\left(w, x_{n}\right)+\sum_{j=1}^{N} \alpha_{n, j} D_{g}\left(x_{n}, x_{n}+e_{n}^{j}\right) \\
& +\sum_{j=1}^{N} \alpha_{n, j}\left\langle w-x_{n}, \nabla g\left(x_{n}\right)-\nabla g\left(x_{n}+e_{n}^{j}\right)\right\rangle \\
& -\alpha_{n, i} \alpha_{n, j} \rho_{s}^{*}\left(\left\|\nabla g\left(T_{i}\left(x_{n}+e_{n}^{i}\right)\right)-\nabla g\left(T_{j}\left(x_{n}+e_{n}^{j}\right)\right)\right\|\right) .
\end{aligned}
$$


Let us show (4.16). For any given $w \in F$ and $j \in\{0,1,2, \ldots, N\}$, in view of the definition of the Bregman distance (see (1.7)), (1.6), Lemmas 2.3 and 2.5, we obtain

$$
\begin{aligned}
& D_{g}\left(w, y_{n}\right)=D_{g}\left(w, \nabla g^{*}\left[\alpha_{n, 0} \nabla g\left(x_{n}\right)+\sum_{j=1}^{N} \alpha_{n, j} \nabla g\left(T_{j}\left(x_{n}+e_{n}^{j}\right)\right)\right]\right) \\
& =V\left(w, \alpha_{n, 0} \nabla g\left(x_{n}\right)+\sum_{j=1}^{N} \alpha_{n, j} \nabla g\left(T_{j}\left(x_{n}+e_{n}^{j}\right)\right)\right) \\
& =g(w)-\left\langle w, \alpha_{n, 0} \nabla g\left(x_{n}\right)+\sum_{j=1}^{N} \alpha_{n, j} \nabla g\left(T_{j}\left(x_{n}+e_{n}^{j}\right)\right)\right\rangle \\
& +g^{*}\left(\alpha_{n, 0} \nabla g\left(x_{n}\right)\right)+\sum_{j=1}^{N} \alpha_{n, j} \nabla g\left(T_{j}\left(x_{n}+e_{n}^{j}\right)\right) \\
& \leq \alpha_{n, 0} g(w)+\sum_{j=1}^{N} \alpha_{n, j} g(w)-\alpha_{n, 0}\left\langle w, \nabla g\left(x_{n}\right)\right\rangle-\sum_{j=1}^{N} \alpha_{n, j}\left\langle w, \nabla g\left(T_{j}\left(x_{n}+e_{n}^{j}\right)\right)\right\rangle \\
& +\alpha_{n, 0} g^{*}\left(\nabla g\left(x_{n}\right)\right)+\sum_{j=1}^{N} \alpha_{n, j} g^{*}\left(\nabla g\left(T_{j}\left(x_{n}+e_{n}^{j}\right)\right)\right) \\
& -\alpha_{n, i} \alpha_{n, j} \rho_{s}^{*}\left(\left\|\nabla g\left(T_{i}\left(x_{n}+e_{n}^{i}\right)\right)-\nabla g\left(T_{j}\left(x_{n}+e_{n}^{j}\right)\right)\right\|\right) \\
& =\alpha_{n, 0} V\left(w, \nabla g\left(x_{n}\right)\right)+\sum_{j=1}^{N} \alpha_{n, j} V\left(w, \nabla g\left(T_{j}\left(x_{n}+e_{n}^{j}\right)\right)\right) \\
& -\alpha_{n, i} \alpha_{n, j} \rho_{s}^{*}\left(\left\|\nabla g\left(T_{i}\left(x_{n}+e_{n}^{i}\right)\right)-\nabla g\left(T_{j}\left(x_{n}+e_{n}^{j}\right)\right)\right\|\right) \\
& =\alpha_{n, 0} D_{g}\left(w, x_{n}\right)+\sum_{j=1}^{N} \alpha_{n, j} D_{g}\left(w, T_{j}\left(x_{n}+e_{n}^{j}\right)\right) \\
& -\alpha_{n, i} \alpha_{n, j} \rho_{s}^{*}\left(\left\|\nabla g\left(T_{i}\left(x_{n}+e_{n}^{i}\right)\right)-\nabla g\left(T_{j}\left(x_{n}+e_{n}^{j}\right)\right)\right\|\right) \\
& \leq \alpha_{n, 0} D_{g}\left(w, x_{n}\right)+\sum_{j=1}^{N} \alpha_{n, j} D_{g}\left(w, x_{n}+e_{n}^{j}\right) \\
& -\alpha_{n, i} \alpha_{n, j} \rho_{s}^{*}\left(\left\|\nabla g\left(T_{i}\left(x_{n}+e_{n}^{i}\right)\right)-\nabla g\left(T_{j}\left(x_{n}+e_{n}^{j}\right)\right)\right\|\right) \\
& =\alpha_{n, 0} D_{g}\left(w, x_{n}\right)+\sum_{j=1}^{N} \alpha_{n, j} D_{g}\left(w, x_{n}\right)+\sum_{j=1}^{N} \alpha_{n, j} D_{g}\left(x_{n}, x_{n}+e_{n}^{j}\right) \\
& +\sum_{j=1}^{N} \alpha_{n, j}\left\langle w-x_{n}, \nabla g\left(x_{n}\right)-\nabla g\left(x_{n}+e_{n}^{j}\right)\right\rangle \\
& -\alpha_{n, i} \alpha_{n, j} \rho_{s}^{*}\left(\left\|\nabla g\left(T_{i}\left(x_{n}+e_{n}^{i}\right)\right)-\nabla g\left(T_{j}\left(x_{n}+e_{n}^{j}\right)\right)\right\|\right) \\
& =D_{g}\left(w, x_{n}\right)+\sum_{j=1}^{N} \alpha_{n, j} D_{g}\left(x_{n}, x_{n}+e_{n}^{j}\right) \\
& +\sum_{j=1}^{N} \alpha_{n, j}\left\langle w-x_{n}, \nabla g\left(x_{n}\right)-\nabla g\left(x_{n}+e_{n}^{j}\right)\right\rangle \\
& -\alpha_{n, i} \alpha_{n, j} \rho_{s}^{*}\left(\left\|\nabla g\left(T_{i}\left(x_{n}+e_{n}^{i}\right)\right)-\nabla g\left(T_{j}\left(x_{n}+e_{n}^{j}\right)\right)\right\|\right) .
\end{aligned}
$$


Since $\lim _{n \rightarrow \infty}\left\|x_{n}-\left(x_{n}+e_{n}^{j}\right)\right\|=0$ for all $j \in\{0,1,2, \ldots, N\}$ and $\nabla g$ is uniformly norm-tonorm continuous on any bounded subset of $E$, we obtain

$$
\lim _{n \rightarrow \infty}\left\|\nabla g\left(x_{n}\right)-\nabla g\left(x_{n}+e_{n}^{j}\right)\right\|=0, \quad \forall j \in\{0,1,2, \ldots, N\}
$$

This, together with (4.15), implies that

$$
\begin{aligned}
& D_{g}\left(w, x_{n}\right)-D_{g}\left(w, y_{n}\right)+\sum_{j=1}^{N} \alpha_{n, j} D_{g}\left(x_{n}, x_{n}+e_{n}^{j}\right) \\
& \quad+\sum_{j=1}^{N} \alpha_{n, j}\left\langle w-x_{n}, \nabla g\left(x_{n}\right)-\nabla g\left(x_{n}+e_{n}^{j}\right)\right\rangle \rightarrow 0 \quad \text { as } n \rightarrow \infty .
\end{aligned}
$$

In view of (4.16) and (4.17), we conclude that

$$
\begin{aligned}
& \alpha_{n, i} \alpha_{n, j} \rho_{s}^{*}\left(\left\|\nabla g\left(T_{i}\left(x_{n}+e_{n}^{i}\right)\right)-\nabla g\left(T_{j}\left(x_{n}+e_{n}^{j}\right)\right)\right\|\right) \\
& \leq D_{g}\left(w, x_{n}\right)-D_{g}\left(w, y_{n}\right)+\sum_{j=1}^{N} \alpha_{n, j} D_{g}\left(x_{n}, x_{n}+e_{n}^{j}\right) \\
& \quad+\sum_{j=1}^{N} \alpha_{n, j}\left\langle x_{n+1}-x_{n}, \nabla g\left(x_{n}\right)-\nabla g\left(x_{n}+e_{n}^{j}\right)\right\rangle \\
& \rightarrow 0
\end{aligned}
$$

as $n \rightarrow \infty$. From the assumption $\liminf _{n \rightarrow \infty} \alpha_{n, i} \alpha_{n, j}>0, \forall j \in\{0,1,2, \ldots, N\}$, we have

$$
\lim _{n \rightarrow \infty} \rho_{s}^{*}\left(\left\|\nabla g\left(T_{i}\left(x_{n}+e_{n}^{i}\right)\right)-\nabla g\left(T_{j}\left(x_{n}+e_{n}^{j}\right)\right)\right\|\right)=0, \quad \forall j \in\{0,1,2, \ldots, N\}
$$

Therefore, from the property of $\rho_{s}^{*}$, we deduce that

$$
\lim _{n \rightarrow \infty}\left\|\nabla g\left(T_{i}\left(x_{n}+e_{n}^{i}\right)\right)-\nabla g\left(T_{j}\left(x_{n}+e_{n}^{j}\right)\right)\right\|=0, \quad \forall j \in\{0,1,2, \ldots, N\}
$$

Since $\nabla g^{*}$ is uniformly norm-to-norm continuous on bounded subsets of $E^{*}$, we arrive at

$$
\lim _{n \rightarrow \infty}\left\|T_{i}\left(x_{n}+e_{n}^{i}\right)-T_{j}\left(x_{n}+e_{n}^{j}\right)\right\|=0, \quad \forall j \in\{0,1,2, \ldots, N\} .
$$

In particular, for $j=0$, we have

$$
\lim _{n \rightarrow \infty}\left\|T_{i}\left(x_{n}+e_{n}^{i}\right)-x_{n}\right\|=\lim _{n \rightarrow \infty}\left\|T_{i}\left(x_{n}+e_{n}^{i}\right)-\left(x_{n}+e_{n}^{0}\right)\right\|=0 .
$$

This, together with (4.7) and (4.18), implies that

$$
\lim _{n \rightarrow \infty}\left\|T_{j}\left(x_{n}+e_{n}^{j}\right)-x_{n}+e_{n}^{j}\right\|=0, \quad \forall j \in\{0,1,2, \ldots, N\} .
$$

From (4.7), we obtain

$$
\lim _{n \rightarrow \infty}\left\|x_{n}+e_{n}^{j}-u\right\|=0, \quad \forall j \in\{0,1,2, \ldots, N\} .
$$


In view of (4.19) and (4.20), we conclude that $T_{j} u=u, \forall j \in\{0,1,2, \ldots, N\}$. Thus, we have $u \in F$.

Finally, we show that $u=\operatorname{proj}_{F}^{g} x$. From $x_{n}=\operatorname{proj}_{C_{n}}^{g} x$, we conclude that

$$
\left\langle z-x_{n}, \nabla g\left(x_{n}\right)-\nabla g(x)\right\rangle \geq 0, \quad \forall z \in C_{n} .
$$

Since $F \subset C_{n}$ for each $n \in \mathbb{N}$, we obtain

$$
\left\langle z-x_{n}, \nabla g\left(x_{n}\right)-\nabla g(x)\right\rangle \geq 0, \quad \forall z \in F
$$

Letting $n \rightarrow \infty$ in (4.21), we deduce that

$$
\langle z-u, \nabla g(u)-\nabla g(x)\rangle \geq 0, \quad \forall z \in F .
$$

In view of (1.8), we have $u=\operatorname{proj}_{F}^{g} x$, which completes the proof.

Remark 4.1 In Theorem 4.1, we present a strong convergence theorem for Bregman weak relatively nonexpansive mappings with a new algorithm and new control conditions. This is complementary to Reich and Sabach [46, Theorem 2]. It also extends and improves Theorems 1.3, 1.4 and 1.5.

\section{Equilibrium problems}

Let $E$ be a Banach space and let $C$ be a nonempty, closed and convex subset of a reflexive Banach space $E$. Let $f: C \times C \rightarrow \mathbb{R}$ be a bifunction. Consider the following equilibrium problem: Find $\bar{x} \in C$ such that

$$
f(\bar{x}, y) \geq 0, \quad \forall y \in C
$$

In order to solve the equilibrium problem, let us assume that $f: C \times C \rightarrow \mathbb{R}$ satisfies the following conditions [53]:

(A1) $f(x, x)=0$ for all $x \in C$;

(A2) $f$ is monotone, i.e., $f(x, y)+f(y, x) \leq 0$ for all $x, y \in C$;

(A3) $f$ is upper hemi-continuous, i.e., for each $x, y, z \in C$,

$$
\limsup _{t \downarrow 0} f(t z+(1-t) x, y) \leq f(x, y)
$$

(A4) for each $x \in C$, the function $y \longmapsto f(x, y)$ is convex and lower semicontinuous.

The set of solutions of problem (5.1) is denoted by $\mathrm{EP}(f)$.

Let $C$ be a nonempty, closed and convex subset of $E$ and let $g: E \rightarrow \mathbb{R}$ be a Legendre function. For $r>0$, we define a mapping $T_{r}: E \rightarrow C$ as follows:

$$
T_{r}(x)=\left\{z \in C: f(z, y)+\frac{1}{r}\langle y-z, \nabla g(z)-\nabla g(x)\rangle \geq 0 \text { for all } y \in C\right\}
$$

for all $x \in E$.

The following two lemmas were proved in [46]. 
Lemma 5.1 Let $E$ be a reflexive Banach space and let $g: E \rightarrow \mathbb{R}$ be a Legendre function. Let $C$ be a nonempty, closed and convex subset of $E$ and let $f: C \times C \rightarrow \mathbb{R}$ be a bifunction satisfying (A1)-(A4). For $r>0$, let $T_{r}: E \rightarrow C$ be the mapping defined by (5.2). Then $\operatorname{dom}\left(T_{r}\right)=E$.

Lemma 5.2 Let $E$ be a reflexive Banach space and let $g: E \rightarrow \mathbb{R}$ be a convex, continuous and strongly coercive function which is bounded on bounded subsets and uniformly convex on bounded subsets of $E$. Let $C$ be a nonempty, closed and convex subset of $E$ and let $f$ : $C \times C \rightarrow \mathbb{R}$ be a bifunction satisfying (A1)-(A4). For $r>0$, let $T_{r}: E \rightarrow C$ be the mapping defined by (5.2). Then the following statements hold:

(1) $T_{r}$ is single-valued;

(2) $T_{r}$ is a Bregman firmly nonexpansive mapping [46], i.e., for all $x, y \in E$,

$$
\left\langle T_{r} x-T_{r} y, \nabla g\left(T_{r} x\right)-\nabla g\left(T_{r} y\right)\right\rangle \leq\left\langle T_{r} x-T_{r} y, \nabla g(x)-\nabla g(y)\right\rangle
$$

(3) $F\left(T_{r}\right)=\mathrm{EP}(f)$;

(4) $\mathrm{EP}(f)$ is closed and convex;

(5) $T_{r}$ is a Bregman quasi-nonexpansive mapping;

(6) $D_{g}\left(q, T_{r} x\right)+D_{g}\left(T_{r} x, x\right) \leq D_{g}(q, x), \forall q \in F\left(T_{r}\right)$.

Theorem 5.1 Let $E$ be a reflexive Banach space and let $g: E \rightarrow \mathbb{R}$ be a strongly coercive Bregman function which is bounded on bounded subsets and uniformly convex and uniformly smooth on bounded subsets of $E$. Let $f$ be a bifunction from $E \times E$ to $\mathbb{R}$ satisfying (A1)-(A4). Let $N \in \mathbb{N}$ and let $\left\{T_{j}\right\}_{j=1}^{N}$ be a finite family of Bregman weak relatively nonexpansive mappings from $E$ into int $\operatorname{dom} g$ such that $F:=\bigcap_{j=1}^{N} F\left(T_{j}\right)$ is a nonempty subset of E. Suppose in addition that $T_{0}=I$, where $I$ is the identity mapping on E. Suppose that $F \cap \mathrm{EP}(f)$ is a nonempty subset of $E$, where $\mathrm{EP}(f)$ is the set of solutions to the equilibrium problem (5.1). Let $\left\{x_{n}\right\}_{n \in \mathbb{N}}$ be a sequence generated by

$$
\left\{\begin{array}{l}
x_{0}=x \in E \quad \text { chosen arbitrarily, } \\
C_{0}=E, \\
y_{n}=\nabla g^{*}\left[\alpha_{n, 0} \nabla g\left(x_{n}\right)+\sum_{j=1}^{N} \alpha_{n, j} \nabla g\left(T_{j}\left(x_{n}+e_{n}^{j}\right)\right)\right], \\
u_{n} \in E \quad \text { such that } f\left(u_{n}, y\right)+\frac{1}{r_{n}}\left\langle y-u_{n}, \nabla g\left(u_{n}\right)-\nabla g\left(y_{n}\right)\right\rangle \geq 0, \forall y \in E, \\
C_{n+1}=\left\{z \in C_{n}: D_{g}\left(z, u_{n}\right) \leq D_{g}\left(z, x_{n}\right)+\sum_{j=1}^{N} \alpha_{n, j} D_{g}\left(x_{n}, x_{n}+e_{n}^{j}\right)\right. \\
\left.\quad+\sum_{j=1}^{N} \alpha_{n, j}\left\langle z-x_{n}, \nabla g\left(x_{n}\right)-\nabla g\left(x_{n}+e_{n}^{j}\right)\right\rangle\right\}, \\
x_{n+1}=\operatorname{proj}_{C_{n+1}}^{g} x \text { and } n \in \mathbb{N} \cup\{0\},
\end{array}\right.
$$

where $\nabla g$ is the right-hand derivative of $g$. Let $\left\{\alpha_{n, j}: n \in \mathbb{N} \cup\{0\}, j \in\{0,1,2, \ldots, N\}\right\}$ be a sequence in $(0,1)$ satisfying the following control conditions:

(1) $\sum_{j=0}^{N} \alpha_{n, j}=1, \forall n \in \mathbb{N} \cup\{0\}$;

(2) There exists $i \in\{1,2, \ldots, N\}$ such that $\liminf _{n \rightarrow \infty} \alpha_{n, i} \alpha_{n, j}>0, \forall j \in\{0,1,2, \ldots, N\}$. If, for each $j=0,1,2, \ldots, N$, the sequences of errors $\left\{e_{n}^{j}\right\}_{n \in \mathbb{N}} \subset E$ satisfy $\liminf _{n \rightarrow \infty} e_{n}^{j}=0$; then the sequence $\left\{x_{n}\right\}_{n \in \mathbb{N}}$ defined in (5.3) converges strongly to $\operatorname{proj}_{F \cap \operatorname{EP}(f)}^{g} x$ as $n \rightarrow \infty$.

Proof By the same argument, as in the proof of Theorem 4.1, we can prove the following: 
(i) $\lim _{n \rightarrow \infty}\left\|x_{n}-u_{n}\right\|=0$ and $\lim _{n \rightarrow \infty}\left\|\nabla g\left(x_{n}\right)-\nabla g\left(u_{n}\right)\right\|=0$.

(ii) For each $w \in F, \lim _{n \rightarrow \infty}\left|D_{g}\left(w, x_{n}\right)-D_{g}\left(w, u_{n}\right)\right|=0$.

(iii) There exists $u \in F$ such that $x_{n} \rightarrow u$ as $n \rightarrow \infty$.

Since $u_{n}=T_{r_{n}} y_{n}$, for any $w \in F$, we have

$$
\begin{aligned}
D_{g}\left(w, u_{n}\right)= & D_{g}\left(w, T_{r_{n}} y_{n}\right) \\
\leq & D_{g}\left(w, y_{n}\right) \\
\leq & D_{g}\left(w, x_{n}\right)+\sum_{j=1}^{N} \alpha_{n, j} D_{g}\left(x_{n}, x_{n}+e_{n}^{j}\right) \\
& +\sum_{j=1}^{N} \alpha_{n, j}\left\langle w-x_{n}, \nabla g\left(x_{n}\right)-\nabla g\left(x_{n}+e_{n}^{j}\right)\right\rangle .
\end{aligned}
$$

Next, we show that $u \in \operatorname{EP}(f)$. From Lemma 5.2(6), (5.4) and $u_{n}=T_{r_{n}} y_{n}$, we conclude that

$$
\begin{aligned}
D_{g}\left(u_{n}, y_{n}\right)= & D_{g}\left(T_{r_{n}} y_{n}, y_{n}\right) \\
\leq & D_{g}\left(w, y_{n}\right)-D_{g}\left(w, T_{r_{n}} y_{n}\right) \\
\leq & D_{g}\left(w, x_{n}\right)-D_{g}\left(w, u_{n}\right)+\sum_{j=1}^{N} \alpha_{n, j} D_{g}\left(x_{n}, x_{n}+e_{n}^{j}\right) \\
& +\sum_{j=1}^{N} \alpha_{n, j}\left\langle w-x_{n}, \nabla g\left(x_{n}\right)-\nabla g\left(x_{n}+e_{n}^{j}\right)\right\rangle \\
\rightarrow & 0
\end{aligned}
$$

as $n \rightarrow \infty$. In view of (5.5) and Lemma 2.4, we obtain

$$
\lim _{n \rightarrow \infty}\left\|u_{n}-y_{n}\right\|=0
$$

Since $\nabla g$ is uniformly norm-to-norm continuous on any bounded subset of $E$, it follows from (5.6) that

$$
\lim _{n \rightarrow \infty}\left\|\nabla g\left(u_{n}\right)-\nabla g\left(y_{n}\right)\right\|=0 .
$$

By the assumption $r_{n} \geq a$, we have

$$
\lim _{n \rightarrow \infty} \frac{\left\|\nabla g\left(u_{n}\right)-\nabla g\left(y_{n}\right)\right\|}{r_{n}}=0 .
$$

In view of $u_{n}=T_{r_{n}} y_{n}$, we obtain

$$
f\left(u_{n}, y\right)+\frac{1}{r_{n}}\left\langle y-u_{n}, \nabla g\left(u_{n}\right)-\nabla g\left(y_{n}\right)\right\rangle \geq 0, \quad \forall y \in E .
$$


From condition (A2), we deduce that

$$
\begin{aligned}
\left\|y-u_{n}\right\| \frac{\left\|\nabla g\left(u_{n}\right)-\nabla g\left(y_{n}\right)\right\|}{r_{n}} & \geq \frac{1}{r_{n}}\left\langle y-u_{n}, \nabla g\left(u_{n}\right)-\nabla g\left(y_{n}\right)\right\rangle \\
& \geq-f\left(u_{n}, y\right) \geq f\left(y, u_{n}\right) \geq 0, \quad \forall y \in E .
\end{aligned}
$$

Letting $n \rightarrow \infty$ in the above inequality, we have from (5.7) and (A4) that

$$
f(y, u) \leq 0, \quad \forall y \in E
$$

For $t \in(0,1]$ and $y \in E$, let $y_{t}=t y+(1-t) u$. Then we have $y_{t} \in E$, which yields that $f\left(y_{t}, u\right) \leq 0$. From (A1), we also have

$$
0=f\left(y_{t}, y_{t}\right) \leq t f\left(y_{t}, y\right)+(1-t) f\left(y_{t}, u\right) \leq t f\left(y_{t}, y\right) .
$$

Dividing by $t$, we get

$$
f\left(y_{t}, y\right) \geq 0, \quad \forall y \in E
$$

Letting $t \downarrow 0$, from the condition (A3), we obtain that

$$
f(u, y) \geq 0, \quad \forall y \in E .
$$

This means that $u \in \operatorname{EP}(f)$. Therefore, $u \in F \cap \operatorname{EP}(f)$.

Theorem 5.2 Let E be a 2-uniformly convex Banach space and let $g: E \rightarrow \mathbb{R}$ be a strongly coercive Bregman function which is bounded on bounded subsets and uniformly convex and uniformly smooth on bounded subsets of $E$. Assume that there exists $c_{1}>0$ such that $g$ is $\rho$-convex with $\rho(t):=\frac{c_{1}}{2} t^{2}$ for all $t \geq 0$. Let $C$ be a nonempty, closed and convex subset of $E$ and let $f$ be a bifunction from $C \times C$ to $\mathbb{R}$ satisfying (A1)-(A4). Assume that $\left\{T_{j}\right\}_{j \in \mathbb{N}}$ is an infinite family of Bregman weak relatively nonexpansive mappings from $C$ into itself and that $A: C \rightarrow E^{*}$ is a $\gamma$-inverse strongly monotone mapping for some $\gamma>0$. Suppose that $F:=\bigcap_{j=1}^{\infty} F\left(T_{j}\right) \cap A^{-1}(0) \cap \mathrm{EP}(f)$ is a nonempty subset of $C$, where $\mathrm{EP}(f)$ is the set of solutions to the equilibrium problem (5.1). Suppose in addition that $T_{0}=I$, where $I$ is the identity mapping on E. Let $\left\{x_{n}\right\}_{n \in \mathbb{N}}$ be a sequence generated by

$$
\left\{\begin{array}{l}
x_{0}=x \in C \quad \text { chosen arbitrarily, } \\
C_{0}=C, \\
y_{n}=\operatorname{proj}_{C}^{g}\left(\nabla g^{*}\left[\nabla g\left(x_{n}\right)-\beta A x_{n}\right]\right), \\
z_{n}=\nabla g^{*}\left[\alpha_{n, 0} \nabla g\left(x_{n}\right)+\sum_{j=1}^{\infty} \alpha_{n, j} \nabla g\left(T_{j} y_{n}\right)\right], \\
u_{n} \in C \quad \text { such that } f\left(u_{n}, y\right)+\frac{1}{r_{n}}\left\langle y-u_{n}, \nabla g\left(u_{n}\right)-\nabla g\left(y_{n}\right)\right\rangle \geq 0, \forall y \in C, \\
C_{n+1}=\left\{z \in C_{n}: D_{g}\left(z, u_{n}\right) \leq D_{g}\left(z, x_{n}\right)\right\}, \\
x_{n+1}=\operatorname{proj}_{C_{n+1}}^{g} x \text { and } n \in \mathbb{N} \cup\{0\},
\end{array}\right.
$$

where $\nabla g$ is the right-hand derivative of $g$. Let $\beta$ be a constant such that $0<\beta<\frac{c_{2}^{2} \gamma}{2}$, where $c_{2}$ is the 2-uniformly convex constant of E satisfying Corollary 2.1(2). Let $\left\{\alpha_{n, j}: n \in \mathbb{N} \cup\{0\}, j \in\right.$ $\mathbb{N} \cup\{0\}\}$ be a sequence in $(0,1)$ satisfying the following control conditions: 
(1) $\sum_{j=0}^{\infty} \alpha_{n, j}=1, \forall n \in \mathbb{N} \cup\{0\}$;

(2) $\liminf _{n \rightarrow \infty} \alpha_{n, 0} \alpha_{n, j}>0, \forall j \in \mathbb{N}$.

Then the sequence $\left\{x_{n}\right\}_{n \in \mathbb{N}}$ defined in (5.8) converges strongly to $\operatorname{proj}_{F}^{g} x$ as $n \rightarrow \infty$.

Proof We divide the proof into several steps.

Step 1. Following the method of the proof of Theorem 3.1 Step 1, we obtain that $C_{n}$ is both closed and convex for each $n \in \mathbb{N} \cup\{0\}$.

Step 2. We claim that $F \subset C_{n}$ for all $n \in \mathbb{N} \cup\{0\}$.

It is obvious that $F \subset C_{0}=C$. Assume now that $F \subset C_{m}$ for some $m \in \mathbb{N}$. It follows from Lemma 2.5 that, for each $w \in F \subset C_{m}$, we have

$$
\begin{aligned}
D_{g}\left(w, y_{m}\right) & =D_{g}\left(w, \operatorname{proj}_{C}^{g}\left(\nabla g^{*}\left[\nabla g\left(x_{m}\right)-\beta A x_{m}\right]\right)\right) \\
& \leq D_{g}\left(w, \nabla g^{*}\left[\nabla g\left(x_{m}\right)-\beta A x_{m}\right]\right) \\
& =V\left(w, \nabla g\left(x_{m}\right)-\beta A x_{m}\right) \\
& \leq V\left(w, \nabla g\left(x_{m}\right)-\beta A x_{m}+\beta A x_{m}\right)-\left\langle\nabla g^{*}\left(\nabla g\left(x_{m}\right)-\beta A x_{m}\right)-w, \beta A x_{m}\right\rangle \\
& =V\left(w, \nabla g\left(x_{m}\right)\right)-\beta\left\langle\nabla g^{*}\left(\nabla g\left(x_{m}\right)-\beta A x_{m}\right)-w, A x_{m}\right\rangle \\
& =D_{g}\left(w, x_{m}\right)-\beta\left\langle x_{m}-w, A x_{m}\right\rangle-\beta\left\langle\nabla g^{*}\left(\nabla g\left(x_{m}\right)-\beta A x_{m}\right)-x_{m}, A x_{m}\right\rangle \\
& \leq D_{g}\left(w, x_{m}\right)-\beta \gamma\left\|A x_{m}\right\|^{2}+\beta\left\|\nabla g^{*}\left(\nabla g\left(x_{m}\right)-\beta A x_{m}\right)-\nabla g^{*} \nabla g\left(x_{m}\right)\right\|\left\|A x_{m}\right\| \\
& \leq D_{g}\left(w, x_{m}\right)-\beta \gamma\left\|A x_{m}\right\|^{2}+\frac{4 \beta^{2}}{c_{2}^{2}}\left\|A x_{m}\right\|^{2} \\
& \leq D_{g}\left(w, x_{m}\right)+\beta\left(\frac{4 \beta}{c_{2}^{2}}-\gamma\right)\left\|A x_{m}\right\|^{2} .
\end{aligned}
$$

This, together with $\frac{4 \beta}{c_{2}^{2}}-\gamma<0$, implies that

$$
D_{g}\left(w, y_{m}\right) \leq D_{g}\left(w, x_{m}\right)
$$

Since $T_{j}$ is Bregman weak relatively nonexpansive, for each $j \in \mathbb{N}$, we obtain

$$
\begin{aligned}
D_{g}\left(w, u_{m}\right)= & D_{g}\left(w, T_{r_{m}} z_{m}\right) \\
\leq & D_{g}\left(w, z_{m}\right) \\
= & D_{g}\left(w, \nabla g^{*}\left[\alpha_{m, 0} \nabla g\left(x_{m}\right)+\sum_{j=1}^{\infty} \alpha_{m, j} \nabla g\left(T_{j} y_{m}\right)\right]\right) \\
= & V\left(w, \alpha_{m, 0} \nabla g\left(x_{m}\right)+\sum_{j=1}^{\infty} \alpha_{m, j} \nabla g\left(T_{j} y_{m}\right)\right) \\
= & g(w)-\left\langle w, \alpha_{m, 0} \nabla g\left(x_{m}\right)+\sum_{j=1}^{\infty} \alpha_{m, j} \nabla g\left(T_{j} y_{m}\right)\right\rangle \\
& +g^{*}\left(\alpha_{m, 0} \nabla g\left(x_{m}\right)+\sum_{j=1}^{\infty} \alpha_{m, j} \nabla g\left(T_{j} y_{m}\right)\right)
\end{aligned}
$$




$$
\begin{aligned}
\leq & \alpha_{m, 0} g(w)+\sum_{j=1}^{\infty} \alpha_{m, j} g(w) \\
& +\alpha_{m, 0} g^{*}\left(\nabla g\left(x_{m}\right)\right)+\sum_{j=1}^{\infty} \alpha_{m, j} g^{*}\left(\nabla g\left(T_{j} y_{m}\right)\right) \\
= & \alpha_{m, 0} V\left(w, \nabla g\left(x_{m}\right)\right)+\sum_{j=1}^{\infty} \alpha_{m, j} V\left(w, \nabla g\left(T_{j} y_{m}\right)\right) \\
= & \alpha_{m, 0} D_{g}\left(w, x_{m}\right)+\sum_{j=1}^{\infty} \alpha_{m, j} D_{g}\left(w, T_{j} y_{m}\right) \\
\leq & \alpha_{m, 0} D_{g}\left(w, x_{m}\right)+\sum_{j=1}^{\infty} \alpha_{m, j} D_{g}\left(w, y_{m}\right) \\
\leq & D_{g}\left(w, x_{m}\right) .
\end{aligned}
$$

This proves that $w \in C_{m+1}$. Consequently, we see that $F \subset C_{n}$ for any $n \in \mathbb{N} \cup\{0\}$.

Step 3. By the same manner, as mentioned in the proof of Theorem 3.1, Step 3, we can prove that the sequences $\left\{x_{n}\right\}_{n \in \mathbb{N}},\left\{y_{n}\right\}_{n \in \mathbb{N}},\left\{z_{n}\right\}_{n \in \mathbb{N}},\left\{u_{n}\right\}_{n \in \mathbb{N}}$ and $\left\{T_{j} y_{n}: j \in \mathbb{N} \cup\{0\}, n \in \mathbb{N} \cup\right.$ $\{0\}\}$ are bounded.

Step 4. We show that $x_{n} \rightarrow u$ for some $u \in F$, where $u=\operatorname{proj}_{F}^{g} x$.

A similar argument, as mentioned in Theorem 3.1, Step 4, shows that there exists $u \in C$ such that

$$
\lim _{n \rightarrow \infty}\left\|x_{n}-u\right\|=0 \text { and } \lim _{n \rightarrow \infty}\left\|u_{n}-x_{n}\right\|=0 .
$$

In view of Lemma 2.4, we deduce that

$$
\lim _{n \rightarrow \infty} D_{g}\left(u_{n}, x_{n}\right)=0
$$

Since $\nabla g$ is uniformly norm-to-norm continuous on any bounded subset of $E$, we obtain

$$
\lim _{n \rightarrow \infty}\left\|\nabla g\left(u_{n}\right)-\nabla g\left(x_{n}\right)\right\|=0
$$

It follows from the three point identity (see (2.2)) that

$$
\begin{aligned}
\left|D_{g}\left(w, x_{n}\right)-D_{g}\left(w, u_{n}\right)\right|= & \mid D_{g}\left(w, u_{n}\right)+D_{g}\left(u_{n}, x_{n}\right) \\
& +\left\langle w-u_{n}, \nabla g\left(u_{n}\right)-\nabla g\left(x_{n}\right)\right\rangle-D_{g}\left(w, u_{n}\right) \mid \\
= & \left|D_{g}\left(u_{n}, x_{n}\right)-\left\langle w-u_{n}, \nabla g\left(u_{n}\right)-\nabla g\left(x_{n}\right)\right\rangle\right| \\
\leq & D_{g}\left(u_{n}, x_{n}\right)+\left\|w-u_{n}\right\|\left\|\nabla g\left(u_{n}\right)-\nabla g\left(x_{n}\right)\right\| \\
\rightarrow & 0
\end{aligned}
$$

as $n \rightarrow \infty$. The function $g$ is bounded on bounded subsets of $E$ and thus $\nabla g$ is also bounded on bounded subsets of $E^{*}$ (see, for example, [23, Proposition 1.1.11] for more details). This, together with Step 3, implies that the sequences $\left\{\nabla g\left(x_{n}\right)\right\}_{n \in \mathbb{N}},\left\{\nabla g\left(y_{n}\right)\right\}_{n \in \mathbb{N}}$, $\left\{\nabla g\left(z_{n}\right)\right\}_{n \in \mathbb{N}}$ and $\left\{\nabla g\left(T_{j} x_{n}\right): j \in \mathbb{N} \cup\{0\}, n \in \mathbb{N} \cup\{0\}\right\}$ are bounded in $E^{*}$. 
In view of Theorem 2.2(3), we know that $\operatorname{dom} g^{*}=E^{*}$ and $g^{*}$ is strongly coercive and uniformly convex on bounded subsets. Let $s=\sup \left\{\left\|\nabla g\left(x_{n}\right)\right\|,\left\|\nabla g\left(T_{j} x_{n}\right)\right\|: j \in \mathbb{N} \cup\{0\}, n \in\right.$ $\mathbb{N} \cup\{0\}\}$ and $\rho_{s}^{*}: E^{*} \rightarrow \mathbb{R}$ be the gauge of uniform convexity of the conjugate function $g^{*}$. For any given $w \in F(T)$ and $j \in \mathbb{N}$, in view of the definition of the Bregman distance (see (1.7)), (1.6), Lemmas 2.3 and 2.5, we obtain

$$
\begin{aligned}
& D_{g}\left(w, u_{n}\right)=D_{g}\left(w, T_{r_{n}} z_{n}\right) \\
& \leq D_{g}\left(w, z_{n}\right) \\
& =D_{g}\left(w, \nabla g^{*}\left[\alpha_{n, 0} \nabla g\left(x_{n}\right)+\sum_{j=1}^{\infty} \alpha_{n, j} \nabla g\left(T_{j} y_{n}\right)\right]\right) \\
& =V\left(w, \alpha_{n, 0} \nabla g\left(x_{n}\right)+\sum_{j=1}^{\infty} \alpha_{n, j} \nabla g\left(T_{j} y_{n}\right)\right) \\
& =g(w)-\left\langle w, \alpha_{n, 0} \nabla g\left(x_{n}\right)+\sum_{j=1}^{\infty} \alpha_{n, j} \nabla g\left(T_{j} y_{n}\right)\right\rangle \\
& +g^{*}\left(\alpha_{n, 0} \nabla g\left(x_{n}\right)+\sum_{j=1}^{\infty} \alpha_{n, j} \nabla g\left(T_{j} y_{n}\right)\right) \\
& \leq \alpha_{n, 0} g(w)+\sum_{j=1}^{\infty} \alpha_{n, j} g(w) \\
& -\alpha_{n, 0}\left\langle w, \nabla g\left(x_{n}\right)\right\rangle-\sum_{j=1}^{\infty} \alpha_{n, j}\left\langle w, \nabla g\left(T_{j} y_{n}\right)\right\rangle \\
& +\alpha_{n, 0} g^{*}\left(\nabla g\left(x_{n}\right)\right)+\sum_{j=1}^{\infty} \alpha_{n, j} g^{*}\left(\nabla g\left(T_{j} y_{n}\right)\right) \\
& -\alpha_{n, i} \alpha_{n, j} \rho_{s}^{*}\left(\left\|\nabla g\left(x_{n}\right)-\nabla g\left(T_{j} y_{n}\right)\right\|\right) \\
& =\alpha_{n, 0} V\left(w, \nabla g\left(x_{n}\right)\right)+\sum_{j=1}^{\infty} \alpha_{n, j} V\left(w, \nabla g\left(T_{j} y_{n}\right)\right) \\
& -\alpha_{n, 0} \alpha_{n, j} \rho_{s}^{*}\left(\left\|\nabla g\left(x_{n}\right)-\nabla g\left(T_{j} y_{n}\right)\right\|\right) \\
& =\alpha_{n, 0} D_{g}\left(w, x_{n}\right)+\sum_{j=1}^{\infty} \alpha_{n, j} D_{g}\left(w, T_{j} y_{n}\right) \\
& -\alpha_{n, 0} \alpha_{n, j} \rho_{s}^{*}\left(\left\|\nabla g\left(x_{n}\right)-\nabla g\left(T_{j} y_{n}\right)\right\|\right) \\
& =\alpha_{n, 0} D_{g}\left(w, x_{n}\right)+\sum_{j=1}^{\infty} \alpha_{n, j} D_{g}\left(w, y_{n}\right) \\
& -\alpha_{n, 0} \alpha_{n, j} \rho_{s}^{*}\left(\left\|\nabla g\left(x_{n}\right)-\nabla g\left(T_{j} y_{n}\right)\right\|\right) \\
& \leq \alpha_{n, 0} D_{g}\left(w, x_{n}\right)+\sum_{j=1}^{\infty} \alpha_{n, j} D_{g}\left(w, x_{n}\right) \\
& -\alpha_{n, 0} \alpha_{n, j} \rho_{s}^{*}\left(\left\|\nabla g\left(x_{n}\right)-\nabla g\left(T_{j} y_{n}\right)\right\|\right) \\
& =D_{g}\left(w, x_{n}\right)-\alpha_{n, 0} \alpha_{n, j} \rho_{s}^{*}\left(\left\|\nabla g\left(x_{n}\right)-\nabla g\left(T_{j} x_{n}\right)\right\|\right) \text {. }
\end{aligned}
$$


In view of (5.10), (5.11) and (5.12), we conclude that

$$
\begin{aligned}
& \alpha_{n, 0} \alpha_{n, j} \rho_{s}^{*}\left(\left\|\nabla g\left(x_{n}\right)-\nabla g\left(T_{j} y_{n}\right)\right\|\right) \\
& \quad \leq D_{g}\left(w, x_{n}\right)-D_{g}\left(w, z_{n}\right) \\
& \quad \rightarrow 0
\end{aligned}
$$

as $n \rightarrow \infty$. From the assumption $\liminf _{n \rightarrow \infty} \alpha_{n, 0} \alpha_{n, j}>0, \forall j \in \mathbb{N}$, we have

$$
\lim _{n \rightarrow \infty} \rho_{s}^{*}\left(\left\|\nabla g\left(x_{n}\right)-\nabla g\left(T_{j} y_{n}\right)\right\|\right)=0, \quad \forall j \in \mathbb{N}
$$

Therefore, from the property of $\rho_{s}^{*}$, we deduce that

$$
\lim _{n \rightarrow \infty}\left\|\nabla g\left(x_{n}\right)-\nabla g\left(T_{j} y_{n}\right)\right\|=0, \quad \forall j \in \mathbb{N}
$$

Since $\nabla g^{*}$ is uniformly norm-to-norm continuous on bounded subsets of $E^{*}$, we arrive at

$$
\lim _{n \rightarrow \infty}\left\|x_{n}-T_{j} y_{n}\right\|=0, \quad \forall j \in \mathbb{N}
$$

Using inequalities (5.9) and (5.12), we obtain

$$
\begin{aligned}
D_{g}\left(w, u_{n}\right) & \leq \alpha_{n, 0} D_{g}\left(w, x_{n}\right)+\sum_{j=1}^{\infty} \alpha_{n, j} D_{g}\left(w, y_{n}\right) \\
& \leq \alpha_{n, 0} D_{g}\left(w, x_{n}\right)+\sum_{j=1}^{\infty} \alpha_{n, j}\left[D_{g}\left(w, x_{n}\right)+\beta\left(\frac{4 \beta}{c_{2}^{2}}-\gamma\right)\left\|A x_{n}\right\|^{2}\right] \\
& =D_{g}\left(w, x_{n}\right)+\beta \sum_{j=1}^{\infty} \alpha_{n, j}\left(\frac{4 \beta}{c_{2}^{2}}-\gamma\right)\left\|A x_{n}\right\|^{2} .
\end{aligned}
$$

It follows from (5.14) that

$$
\beta \sum_{j=1}^{\infty} \alpha_{n, j}\left(\gamma-\frac{4 \beta}{c_{2}^{2}}\right)\left\|A x_{n}\right\|^{2} \leq D_{g}\left(w, x_{n}\right)-D_{g}\left(w, u_{n}\right) .
$$

Since $\frac{4 \beta}{c_{2}^{2}}-\gamma<0$, we see that

$$
\lim _{n \rightarrow \infty}\left\|A x_{n}\right\|=0
$$

Furthermore, since $x_{n} \in C$ for all $n \geq 0$, then using (1.6), Lemma 2.5 and Corollary 2.1, we get

$$
\begin{aligned}
D_{g}\left(x_{n}, y_{n}\right) & =D_{g}\left(x_{n}, \operatorname{proj}_{C}^{g}\left(\nabla g^{*}\left[\nabla g\left(x_{n}\right)-\beta A x_{n}\right]\right)\right) \\
& \leq D_{g}\left(x_{n}, \nabla g^{*}\left[\nabla g\left(x_{n}\right)-\beta A x_{n}\right]\right) \\
& =V\left(x_{n}, \nabla g\left(x_{n}\right)-\beta A x_{n}\right) \\
& \leq V\left(x_{n}, \nabla g\left(x_{n}\right)-\beta A x_{n}+\beta A x_{n}\right)-\left\langle\nabla g^{*}\left(\nabla g\left(x_{n}\right)-\beta A x_{n}\right)-x_{n}, \beta A x_{n}\right\rangle
\end{aligned}
$$




$$
\begin{aligned}
& =V\left(x_{n}, \nabla g\left(x_{n}\right)\right)-\beta\left\langle\nabla g^{*}\left(\nabla g\left(x_{n}\right)-\beta A x_{n}\right)-w, A x_{n}\right\rangle \\
& =D_{g}\left(x_{n}, x_{n}\right)-\beta\left\langle x_{n}-x_{n}, A x_{n}\right\rangle-\beta\left\langle\nabla g^{*}\left(\nabla g\left(x_{n}\right)-\beta A x_{n}\right)-x_{n}, A x_{n}\right\rangle \\
& \leq \beta\left\|\nabla g^{*}\left(\nabla g\left(x_{n}\right)-\beta A x_{n}\right)-\nabla g^{*} \nabla g\left(x_{n}\right)\right\|\left\|A x_{n}\right\| \\
& \leq \frac{4 \beta^{2}}{c_{2}^{2}}\left\|A x_{n}\right\|^{2} .
\end{aligned}
$$

It follows from (5.15) that

$$
\lim _{n \rightarrow \infty} D_{g}\left(x_{n}, y_{n}\right)=0
$$

Lemma 2.2 now implies that

$$
\lim _{n \rightarrow \infty}\left\|x_{n}-y_{n}\right\|=0
$$

Using (5.13) and (5.16), we conclude that

$$
\lim _{n \rightarrow \infty}\left\|y_{n}-u\right\|=0 \quad \text { and } \quad \lim _{n \rightarrow \infty}\left\|y_{n}-T_{j} y_{n}\right\|=0, \quad \forall j \in \mathbb{N} .
$$

Therefore, $u \in \tilde{F}\left(T_{j}\right)=F\left(T_{j}\right), \forall j \in \mathbb{N}$.

Step 5. We show that $u \in A^{-1}(0)$.

Since $A$ is $\gamma$-inverse strongly monotone, it is continuous and hence, using (5.16) and (5.17), we conclude that $A u=\lim _{n \rightarrow \infty} A x_{n}=0$. Therefore, $u \in A^{-1}(0)$.

Step 6. Finally, we show that $u=\operatorname{proj}_{F}^{g} x$.

The proof of this step is similar to that of Theorem 3.1, Step 4 and is omitted here.

We end this section with the following simple example in order to support Theorem 5.2.

Example 5.1 Let $E=l^{2}$ and

$$
\begin{aligned}
& x_{0}=(1,0,0,0, \ldots), \\
& x_{1}=(1,1,0,0,0, \ldots), \\
& x_{2}=(1,0,1,0,0,0, \ldots), \\
& x_{3}=(1,0,0,1,0,0,0, \ldots), \\
& \ldots, \\
& x_{n}=\left(\sigma_{n, 1}, \sigma_{n, 2}, \ldots, \sigma_{n, k}, \ldots\right), \\
& \ldots,
\end{aligned}
$$

where

$$
\sigma_{n, k}= \begin{cases}1 & \text { if } k=1, n+1, \\ 0 & \text { if } k \neq 1, k \neq n+1\end{cases}
$$


for all $n \in \mathbb{N}$. It is easy to see that the sequence $\left\{x_{n}\right\}_{n \in \mathbb{N}}$ converges weakly to $x_{0}$. Let $k$ be an even number in $\mathbb{N}$ and let $g: E \rightarrow \mathbb{R}$ be defined by

$$
g(x)=\frac{1}{k}\|x\|^{k}, \quad x \in E .
$$

It is easy to show that $\nabla g(x)=J_{k}(x)$ for all $x \in E$, where

$$
J_{k}(x)=\left\{x^{*} \in E^{*}:\left\langle x, x^{*}\right\rangle=\|x\|\left\|x^{*}\right\|,\left\|x^{*}\right\|=\|x\|^{k-1}\right\} .
$$

It is also obvious that

$$
J_{k}(\lambda x)=\lambda^{k-1} J_{k}(x), \quad \forall x \in E, \lambda \in \mathbb{R} .
$$

Now, we define a countable family of mappings $T_{j}: E \rightarrow E$ by

$$
T_{j}(x)= \begin{cases}\frac{n}{n+1} x & \text { if } x=x_{n} \\ \frac{-x}{j} & \text { if } x \neq x_{n}\end{cases}
$$

for all $j \geq 1$ and $n \geq 0$. It is clear that $F\left(T_{j}\right)=\{0\}$ for all $j \geq 1$. Choose $j \in \mathbb{N}$, then for any $n \in \mathbb{N}$,

$$
\begin{aligned}
D_{g}\left(0, T_{j} x_{n}\right) & =g(0)-g\left(T_{j} x_{n}\right)-\left\langle 0-T_{j} x_{n}, \nabla g\left(T_{j} x_{n}\right)\right\rangle \\
& =-\frac{n^{k}}{(n+1)^{k}} g\left(x_{n}\right)+\frac{n^{k}}{(n+1)^{k}}\left\langle x_{n}, \nabla g\left(x_{n}\right)\right\rangle \\
& =\frac{n^{k}}{(n+1)^{k}}\left[-g\left(x_{n}\right)+\left\langle x_{n}, \nabla g\left(x_{n}\right)\right\rangle\right] \\
& =\frac{n^{k}}{(n+1)^{k}}\left[D_{g}\left(0, x_{n}\right)\right] \\
& \leq D_{g}\left(0, x_{n}\right) .
\end{aligned}
$$

If $x \neq x_{n}$, then we have

$$
\begin{aligned}
D_{g}\left(0, T_{j} x\right) & =g(0)-g\left(T_{j} x\right)-\left\langle 0-T_{j} x, \nabla g\left(T_{j} x\right)\right\rangle \\
& =-\frac{1}{j^{k}} g(x)-\frac{1}{j^{k}}\langle x,-\nabla g(x)\rangle \\
& =\frac{1}{j^{k}}[-g(x)-\langle-x, \nabla g(x)\rangle] \\
& \leq D_{g}(0, x) .
\end{aligned}
$$

Therefore, $T_{j}$ is a Bregman quasi-nonexpansive mapping. Next, we claim that $T_{j}$ is a Bregman weak relatively nonexpansive mapping. Indeed, for any sequence $\left\{z_{n}\right\}_{n \in \mathbb{N}} \subset E$ such that $z_{n} \rightarrow z_{0}$ and $\left\|z_{n}-T_{j} z_{n}\right\| \rightarrow 0$ as $n \rightarrow \infty$, there exists a sufficiently large number $N_{0} \in \mathbb{N}$ such that $z_{n} \neq x_{m}$ for any $n, m>N_{0}$. This implies that $T_{j} z_{n}=-\frac{z_{n}}{j}$ for all $n>N_{0}$. It follows from $\left\|z_{n}-T_{j} z_{n}\right\| \rightarrow 0$ that $\frac{j+1}{j} z_{n} \rightarrow 0$ and hence $z_{n} \rightarrow z_{0}=0$. Since $z_{0} \in F\left(T_{j}\right)$, 
we conclude that $T_{j}$ is a Bregman weak relatively nonexpansive mapping. It is clear that $\bigcap_{j=1}^{\infty} \tilde{F}\left(T_{j}\right)=\bigcap_{j=1}^{\infty} F\left(T_{j}\right)=\{0\}$. Thus $\left\{T_{j}\right\}_{j \in \mathbb{N}}$ is a countable family of Bregman weak relatively nonexpansive mappings.

Next, we show that $\left\{T_{j}\right\}_{j \in \mathbb{N}}$ is not a countable family of Bregman relatively nonexpansive mappings. In fact, though $x_{n} \rightarrow x_{0}$ and

$$
\left\|x_{n}-T_{j} x_{n}\right\|=\left\|x_{n}-\frac{n}{n+1} x_{n}\right\|=\frac{1}{n+1}\left\|x_{n}\right\| \rightarrow 0
$$

as $n \rightarrow \infty$, but $x_{0} \notin F\left(T_{j}\right)$ for all $j \in \mathbb{N}$. Therefore, $\hat{F}\left(T_{j}\right) \neq F\left(T_{j}\right)$ for all $j \in \mathbb{N}$. This implies that $\bigcap_{j=1}^{\infty} \hat{F}\left(T_{j}\right) \neq \bigcap_{j=1}^{\infty} F\left(T_{j}\right)$.

Finally, it is obvious that the family $\left\{T_{j}\right\}_{j \in \mathbb{N}}$ satisfies all the aspects of the hypothesis of Theorem 5.2.

\section{Applications (Hammerstein-type equations)}

Let $E$ be a real Banach space with the dual space $E^{*}$. The generalized formulation of many boundary value problems for ordinary and partial differential equations leads to operator equations of the type

$$
\langle z, A x\rangle=\langle z, b\rangle, \quad \forall z \in E,
$$

which is equivalent to equality of functionals on $E$. That is, the equality of the form

$$
A x=b,
$$

where $A$ is a monotone-type operator acting from a Banach space $E$ into $E^{*}$. Without loss of generality, we may assume $b=0$. It is well known that a solution of the equation $A x=0$ (i.e., $\langle z, A x\rangle=0, \forall z \in E$ ) is a solution of the variational inequality $\langle z-x, A x\rangle \geq 0, \forall z \in E$. Therefore, the theory of monotone operators and its applications to nonlinear partial differential equations and variational inequalities are related and have been involved in a substantial topic in nonlinear functional analysis. One important application of solving (6.1) is finding the zeros of the so-called equation of Hammerstein type (see, e.g., [54]), where a nonlinear integral equation of Hammerstein type is one of the form

$$
u(x)+\int_{\Omega} k(x, y) f(y, u(y)) d y=h(x)
$$

where $d y$ is a $\sigma$-finite measure on the measure space $\Omega$; the real kernel $k$ is defined on $\Omega \times \Omega, f$ is a real-valued function defined on $\Omega \times \mathbb{R}$ and is, in general, nonlinear and $h$ is a given function on $\Omega$. If we now define an operator $K$ by $K v(x)=\int_{\Omega} k(x, y) v(y) d y ; x \in \Omega$, and the so-called superposition or Nemytskii operator by $Q u(y):=f(y, u(y))$, then the integral Eq. (6.2) can be put in operator theoretic form as follows:

$$
u+K Q u=0
$$

where, without loss of generality, we have taken $h=0$. 
Interest in Eq. (6.2) stems mainly from the fact that several problems that arise in differential equations, for instance, elliptic boundary value problems, whose linear parts possess Green's functions, can, as a rule, be transformed into equations of the form (6.2) (see, e.g., [55], Chapter IV). Equations of Hammerstein type play a crucial role in the theory of optimal control systems (see, e.g., [56]). Several existence and uniqueness theorems have been proved for equations of Hammerstein type (see, e.g., [57-62]). Very recently, Ofoedu and Malonza in [63] proposed an iterative solution of the operator Hammerstein Eq. (6.1) in a 2-uniformly convex and uniformly smooth Banach space.

Now, we give an application of Theorem 5.1 to an iterative solution of the operator Hammerstein Eq. (6.1).

Theorem 6.1 Let $E$ be a real Banach space with a dual space $E^{*}$ such that $X=E \times E^{*}$ (with the norm $\left.\|z\|_{X}^{2}=\|u\|_{E}^{2}+\|v\|_{E^{*}}^{2}, z=(u, v) \in X\right)$ is a 2-uniformly convex and uniformly smooth real Banach space. Let $g: X \rightarrow \mathbb{R}$ be a strongly coercive Bregman function which is bounded on bounded subsets and uniformly convex and uniformly smooth on bounded subsets of $X$. Assume that there exists $c_{1}>0$ such that $g$ is $\rho$-convex with $\rho(t):=\frac{c_{1}}{2} t^{2}$ for all $t \geq 0$. Let $Q$ : $E \rightarrow E^{*}$ and $K: E^{*} \rightarrow E$ with $\operatorname{dom} K=Q(E)=E^{*}$ be continuous monotone-type operators such that Eq. (6.3) has a solution in $E$ and such that the map $A: X \rightarrow X^{*}$ defined by $A z:=$ $A(u, v)=(Q u-v, u+K v)$ is $\gamma$-inverse strongly monotone. Let $C$ be a nonempty, closed and convex subset of $X$, let $f: C \times C \rightarrow \mathbb{R}$ be a bifunction satisfying (A1)-(A4) and let $\left\{T_{j}\right\}_{j \in \mathbb{N}}$ be an infinite family of Bregman weak relatively nonexpansive mappings from $C$ into itself. Let $\left\{x_{n}\right\}_{n \in \mathbb{N}}$ be a sequence generated by

$$
\left\{\begin{array}{l}
x_{0}=x \in C \quad \text { chosen arbitrarily, } \\
C_{0}=C \\
y_{n}=\operatorname{proj}_{C}^{g}\left(\nabla g^{*}\left[\nabla g\left(x_{n}\right)-\beta A x_{n}\right]\right), \\
z_{n}=\nabla g^{*}\left[\alpha_{n, 0} \nabla g\left(x_{n}\right)+\sum_{j=1}^{\infty} \alpha_{n, j} \nabla g\left(T_{j} y_{n}\right)\right], \\
u_{n} \in C \quad \text { such that } f\left(u_{n}, y\right)+\frac{1}{r_{n}}\left\langle y-u_{n}, \nabla g\left(u_{n}\right)-\nabla g\left(y_{n}\right)\right\rangle \geq 0, \forall y \in C, \\
C_{n+1}=\left\{z \in C_{n}: D_{g}\left(z, u_{n}\right) \leq D_{g}\left(z, x_{n}\right)\right\}, \\
x_{n+1}=\operatorname{proj}_{C_{n+1}}^{g} x \text { and } n \in \mathbb{N} \cup\{0\},
\end{array}\right.
$$

where $\nabla g$ is the right-hand derivative of g. Let $\beta$ be a constant such that $0<\beta<\frac{c_{2}^{2} \gamma}{2}$, where $c_{2}$ is the 2-uniformly convex constant of $E$ satisfying Corollary 2.1(2). Let $\left\{\alpha_{n, j}: n \in \mathbb{N} \cup\{0\}, j \in\right.$ $\mathbb{N} \cup\{0\}\}$ be a sequence in $(0,1)$ satisfying the following control conditions:

(1) $\sum_{j=0}^{\infty} \alpha_{n, j}=1, \forall n \in \mathbb{N} \cup\{0\}$;

(2) $\liminf _{n \rightarrow \infty} \alpha_{n, 0} \alpha_{n, j}>0, \forall j \in \mathbb{N}$.

Suppose that $F:=\bigcap_{j=1}^{\infty} F\left(T_{j}\right) \cap A^{-1}(0) \cap \mathrm{EP}(f) \neq \varnothing$, then the sequence $\left\{x_{n}\right\}_{n \in \mathbb{N}}$ defined by (6.4) converges strongly to $\operatorname{proj}_{F}^{g} x$ as $n \rightarrow \infty$.

Remark 6.1 Observe that $z_{0} \in F$ implies, in particular, that $z_{0} \in A^{-1}(0) \Longleftrightarrow A z_{0}=0$. But $z_{0}=\left(u_{0}, v_{0}\right)$ for some $u_{0} \in E$ and $v_{0} \in E^{*}$; moreover, $A z_{0}=A\left(u_{0}, v_{0}\right)=\left(Q u_{0}-v_{0}, u_{0}+K v_{0}\right)$. So, $A z_{0}=0$ implies that $\left(Q u_{0}-v_{0}, u_{0}+K v_{0}\right)=(0,0)$. This is equivalent to $Q u_{0}-v_{0}=0$ and $u_{0}+K v_{0}=0$. Thus we have $v_{0}=Q u_{0}$ which in turn implies that $u_{0}+K v_{0}=0$. Therefore, $u_{0} \in E$ solves the Hammerstein-type Eq. (6.3). 


\section{Competing interests}

The authors declare that they have no competing interests.

\section{Authors' contributions}

All authors contributed equally to this work. All authors read and approved the final manuscript.

\section{Author details}

${ }^{1}$ Department of Mathematics, Yasouj University, Yasouj, 75918, Iran. ${ }^{2}$ Department of Applied Mathematics, National Sun Yat-sen University, Kaohsiung, 804, Taiwan. ${ }^{3}$ Center for Fundamental Science, Kaohsiung Medical University, Kaohsiung, 807, Taiwan. ${ }^{4}$ Department of Mathematics, King Abdulaziz University, P.O. Box 80203, Jeddah, 21589, Saudi Arabia.

\section{Acknowledgements}

Dedicated to Professor Wataru Takahashi on the occasion of his seventieth birthday.

The work of Eskandar Naraghirad was conducted with a postdoctoral fellowship at the National Sun Yat-sen University of Kaohsing. This research was partially supported by the Grant NSC 99-2115-M-037-002-MY3.

\section{Received: 25 April 2013 Accepted: 9 May 2013 Published: 30 May 2013}

\section{References}

1. Haugazeau, Y: Sur les inéquations variationnelles et la minimisation de fonctionnelles convexes. Thése, Université de Paris, Paris, France (1968)

2. Ibaraki, T, Takahashi, W: Strong convergence theorems for a finite family of nonlinear operators of firmly nonexpansive type in Banach spaces. In: Hsu, S-B, Lai, H-C, Lin, L-J, Takahashi, W, Tanaka, T, Yao, J-C (eds.) Nonlinear Analysis and Convex Analysis, pp. 49-62. Yokahama Publishers, Yokahama (2009)

3. Kim, TH, Takahashi, W: Strong convergence of modified iteration processes for relatively asymptotically nonexpansive mappings. Taiwan. J. Math. 14(6), 2163-2180 (2010)

4. Bauschke, HH, Combettes, PL: A weak-to-strong convergence principle for Fejér-monotone methods in Hilbert spaces. Math. Oper. Res. 26, 248-264 (2011)

5. Plubtieng, S, Ungchittrakool, K: Strong convergence theorems for a common fixed point of two relatively nonexpansive mappings in a Banach space. J. Approx. Theory 149, 103-115 (2007)

6. Qin, X, Cho, YJ, Kang, SM, Zhou, H: Convergence of a modified Halpern-type iteration algorithm for quasi- $\phi$-nonexpansive mappings. Appl. Math. Lett. 22, 1051-1055 (2009)

7. Qin, X, Cho, YJ, Kang, SM: Convergence theorems of common elements for equilibrium problems and fixed point problems in Banach spaces. J. Comput. Appl. Math. 225, 20-30 (2009)

8. Qin, X, Cho, SY, Kang, SM: On hybrid projection methods for asymptotically quasi- $\phi$-nonexpansive mappings. Appl. Math. Comput. 215, 3874-3883 (2010)

9. Qin, $X$, Su, Y: Strong convergence theorem for relatively nonexpansive mappings in a Banach space. Nonlinear Anal. 67, 1958-1965 (2007)

10. Qin, $X, S u, Y$, Shang, M: Strong convergence theorems for asymptotically nonexpansive mappings by hybrid methods. Kyungpook Math. J. 48, 133-142 (2008)

11. Matsushita, S, Takahashi, W: Weak and strong convergence theorems for relatively nonexpansive mappings in Banach spaces. Fixed Point Theory Appl. 2004, 37-47 (2004)

12. Takahashi, W, Zembayashi, K: Strong and weak convergence theorems for equilibrium problems and relatively nonexpansive mappings in Banach spaces. Nonlinear Anal. 70, 45-57 (2009)

13. Takahashi, W, Takeuchi, Y, Kubota, R: Strong convergence theorems by hybrid methods for families of nonexpansive mappings in Hilbert spaces. J. Math. Anal. Appl. 341, 276-286 (2008)

14. Takahashi, W: Nonlinear Functional Analysis, Fixed Point Theory and Its Applications. Yokahama Publishers, Yokahama (2000)

15. Takahashi, W: Convex Analysis and Approximation of Fixed Points. Yokahama Publishers, Yokahama (2000)

16. Mann, WR: Mean value methods in iteration. Proc. Am. Math. Soc. 4, 506-510 (1953)

17. Reich, S: Weak convergence theorems for nonexpansive mappings in Banach spaces. J. Math. Anal. Appl. 67, 274-276 (1979)

18. Genel, A, Lindenstrauss, J: An example concerning fixed points. Isr. J. Math. 22, 81-86 (1975)

19. Güler, O: On the convergence of the proximal point algorithm for convex optimization. SIAM J. Control Optim. 29 403-419 (1991)

20. Alber, Y: Metric and generalized projection operators in Banach spaces: properties and applications. In: Kartsatos, A (ed.) Theory and Applications of Nonlinear Operators of Accretive and Monotone Type, pp. 15-50. Dekker, New York (1996)

21. Reich, S: A weak convergence theorem for the altering method with Bregman distances. In: Theory and Applications of Nonlinear Operators of Accretive and Monotone Type, pp. 313-318. Dekker, New York (1996)

22. Matsushita, S, Takahashi, W: A strong convergence theorem for relatively nonexpansive mappings in a Banach space. J. Approx. Theory 134(2), 257-266 (2005)

23. Butnariu, D, lusem, AN: Totally Convex Functions for Fixed Points Computation and Infinite Dimensional Optimization. Kluwer Academic, Dordrecht (2000)

24. Kohsaka, F, Takahashi, W: Proximal point algorithms with Bregman functions in Banach spaces. J. Nonlinear Convex Anal. 6(3), 505-523 (2005)

25. Rockafellar, RT: Monotone operators and the proximal point algorithm. SIAM J. Control Optim. 14, 877-898 (1976)

26. Rockafellar, RT: On the maximality of sums of nonlinear monotone operators. Trans. Am. Math. Soc. 149, 75-88 (1970)

27. Rockafellar, RT: Characterization of subdifferentials of convex functions. Pac. J. Math. 17, 497-510 (1966)

28. Rockafellar, RT: On the maximal monotonicity of subdifferential mappings. Pac. J. Math. 33, 209-216 (1970)

29. Bauschke, HH, Borwein, JM, Combettes, PL: Essential smoothness, essential strict convexity, and Legendre functions in Banach spaces. Commun. Contemp. Math. 3, 615-647 (2001) 
30. Bonnas, JF, Shapiro, A: Perturbation Analysis of Optimization Problems. Springer, New York (2000)

31. Bauschke, HH, Borwein, JM: Legendre functions and the method of random Bregman functions. J. Convex Anal. 4 27-67 (1997)

32. Bregman, LM: The relation method of finding the common point of convex sets and its application to the solution of problems in convex programming. U.S.S.R. Comput. Math. Math. Phys. 7, 200-217 (1967)

33. Censor, Y, Lent, A: An iterative row-action method for interval convex programming. J. Optim. Theory Appl. 34 321-358 (1981)

34. Naraghirad, E, Takahashi, W, Yao, J-C: Generalized retraction and fixed point theorems using Bregman functions in Banach spaces. J. Nonlinear Convex Anal. 13(1), 141-156 (2012)

35. Zălinescu, C: Convex Analysis in General Vector Spaces. World Scientific, River Edge (2002)

36. Butnariu, D, Censor, Y, Reich, S: Iterative averaging of entropic projections for solving stochastic convex feasibility problems. Comput. Optim. Appl. 8, 21-39 (1997)

37. Butnariu, D, Resmerita, E: Bregman distances, totally convex functions and a method for solving operator equations in Banach spaces. Abstr. Appl. Anal. 2006, Article ID 84919 (2006)

38. Bauschke, HH, Borwein, JM, Combettes, PL: Bregman monotone optimization algorithms. SIAM J. Control Optim. 42, 596-636 (2003)

39. Borwein, MJ, Reich, S, Sabach, S: A characterization of Bregman firmly nonexpansive operators using a new monotonicity concept. J. Nonlinear Convex Anal. 12(1), 161-184 (2011)

40. Reich, S, Sabach, S: Two strong convergence theorems for a proximal method in reflexive Banach spaces. Numer. Funct. Anal. Optim. 31, 22-44 (2010)

41. Su, Y, Wang, Z, Xu, HK: Strong convergence theorems for a common fixed point of two hemi-relatively nonexpansive mappings. Nonlinear Anal. 71, 5616-5628 (2009)

42. Bauschke, $\mathrm{HH}$, Combettes, PL: Construction of best Bregman approximations in reflexive Banach spaces. Proc. Am Math. Soc. 131(12), 3757-3766 (2003)

43. Reich, S, Sabach, S: A projection method for solving nonlinear problems in reflexive Banach spaces. J. Fixed Point Theory Appl. 9, 101-116 (2011)

44. Reich, S, Sabach, S: Existence and approximation of fixed points of Bregman firmly nonexpansive mappings in reflexive Banach spaces. In: Fixed-Point Algorithms for Inverse Problems in Science and Engineering. Springer Optimization and Its Applications, vol. 49, pp. 301-316. Springer, New York (2011)

45. Censor, Y, Reich, S: Iterations of paracontractions and firmly nonexpansive operators with applications to feasibility and optimization. Optimization 37, 323-339 (1996)

46. Reich, S, Sabach, S: Two strong convergence theorems for Bregman strongly nonexpansive operators in reflexive Banach spaces. Nonlinear Anal. 73, 122-135 (2010)

47. Bruck, RE, Reich, S: Nonexpansive projections and resolvents of accretive operators in Banach spaces. Houst. J. Math 3, 459-470 (1977)

48. Reich, S: A limit theorem for projections. Linear Multilinear Algebra 13, 281-290 (1983)

49. Sabach, S: Products of finitely many resolvents of maximal monotone mappings in reflexive Banach spaces. SIAM J. Optim. 21, 1289-1308 (2011)

50. Reich, S, Sabach, S: A strong convergence theorem for a proximal-type algorithm in Banach spaces. J. Nonlinear Convex Anal. 10(3), 471-486 (2009)

51. Chang, SS, Chan, CK, Lee, HWJ: Modified block iterative algorithm for quasi- $\phi$-asymptotically nonexpansive mappings and equilibrium problem in Banach spaces. Appl. Math. Comput. 217(18), 7520-7530 (2011)

52. Nakajo, K, Takahashi, W: Strong convergence theorems for nonexpansive mappings and nonexpansive semigroups. J. Math. Anal. Appl. 279, 372-379 (2003)

53. Blum, E, Oettli, W: From optimization and variational inequalities to equilibrium problems. Math. Stud. 63, 123-145 (1994)

54. Hammerstein, A: Nichtlineare integralgleichungen nebst anwendungen. Acta Math. 54, 117-176 (1930)

55. Pascali, D, Sburlan, S: Nonlinear Mappings of Monotone Type. Editura Academiae, Bucharest (1978)

56. Dolezale, V: Monotone Operators and Its Applications in Automation and Network Theory. Studies in Automation and Control, vol. 3. Elsevier, New York (1979)

57. Browder, FE, de Fiqueiredo, DG, Gupta, P: Maximal monotone operators and nonlinear integral equations of Hammerstein type. Bull. Am. Math. Soc. 76, 700-705 (1970)

58. Browder, FE, Gupta, P: Monotone operators and nonlinear integral equations of Hammerstein type. Bull. Am. Math. Soc. 75, 1347-1353 (1969)

59. Chang, SS: On Chidume's open questions and approximation solutions of multi-valued strongly accretive mapping equations in Banach spaces. J. Math. Anal. Appl. 216, 94-111 (1997)

60. Chepanovich, RS: Nonlinear Hammerstein equations and fixed points. Publ. Inst. Math. (Belgr.) 35, 119-123 (1984)

61. Chidume, CE: Fixed point iterations for nonlinear Hammerstein equations involving nonexpansive and accretive mappings. Indian J. Pure Appl. Math. 120, 129-135 (1989)

62. de Figueiredo, DG, Gupta, CP: On the variational method for the existence of solutions to nonlinear equations of Hammerstein type. Proc. Am. Math. Soc. 40, 470-476 (1973)

63. Ofoedu, EU, Malonza, DM: Hybrid approximation of solutions of nonlinear operator equations and applications to equation of Hammerstein-type. Appl. Math. Comput. 217, 6019-6030 (2011)

doi:10.1186/1687-1812-2013-141

Cite this article as: Naraghirad and Yao: Bregman weak relatively nonexpansive mappings in Banach spaces. Fixed Point Theory and Applications 2013 2013:141. 